\title{
The energetics of relativistic magnetic reconnection: ion-electron repartition and particle distribution hardness
}

\author{
Mickaël Melzani ${ }^{1}$, Rolf Walder ${ }^{1}$, Doris Folini ${ }^{1}$, Christophe Winisdoerffer ${ }^{1}$, and Jean M. Favre ${ }^{2}$ \\ ${ }^{1}$ École Normale Supérieure, Lyon, CRAL, UMR CNRS 5574, Université de Lyon, France \\ E-mail: mickael.melzani@ens-lyon.fr \\ 2 CSCS Lugano, Switzerland
}

Received 12 May 2014 / Accepted 18 August 2014

ABSTRACT

\begin{abstract}
Collisionless magnetic reconnection is a prime candidate to account for flare-like or steady emission, outflow launching, or plasma heating, in a variety of high-energy astrophysical objects, including ones with relativistic ion-electron plasmas. But the fate of the initial magnetic energy in a reconnection event remains poorly known. What are the amounts assigned to kinetic energy, the ion and electron distribution, and the hardness of the particle distributions? We explored these questions with 2D particle-in-cell simulations of ion-electron plasmas. We find that 45 to $75 \%$ of the total initial magnetic energy ends up in kinetic energy, this fraction increasing with the inflow magnetization. Depending on the guide field strength, ions get from $30 \%$ to $60 \%$ of the total kinetic energy. Particles can be separated into two populations that mix only weakly: (i) particles initially in the current sheet heated by its initial tearing and subsequent contraction of the islands, and (ii) particles from the background plasma that primarily gain energy via the reconnection electric field when passing near the X-point. Particles of (ii) tend to form a power law with an index $p=-\operatorname{dlog} n(\gamma) / \mathrm{d} \log \gamma$ that depends mostly on the inflow Alfvén speed $V_{\mathrm{A}}$ and magnetization $\sigma_{s}$ of species $s$. For electrons $p=5$ to 1.2 for increasing $\sigma_{\mathrm{e}}$. The highest particle Lorentz factor for ions or electrons increases roughly linearly with time for all the relativistic simulations. This is faster, and the spectra can be harder, than for collisionless shock acceleration. We discuss applications to microquasar and AGN coronae, to extragalactic jets, and to radio lobes. We point out situations where effects, such as Compton drag or pair creation, are important.
\end{abstract}

Key words. plasmas - methods: numerical - magnetic reconnection - instabilities - relativistic processes

\section{Introduction}

Magnetic reconnection is a prime mechanism invoked to produce high-energy particles, radiation, and high-energy flares, to launch large scale outflows, or to efficiently heat plasmas in a variety of astrophysical objects. It is a candidate for explaining (i) particle acceleration at pulsar wind termination shocks (Kirk \& Skjæraasen 2003; Pétri \& Lyubarsky 2007; Sironi \& Spitkovsky 2011a); (ii) the flat radio spectra from galactic nuclei and AGNs (Birk et al. 2001) and from extragalactic jets (Romanova \& Lovelace 1992); (iii) $\mathrm{GeV}$ flares from the Crab nebula (Bednarek \& Idec 2011; Uzdensky et al. 2011; Cerutti et al. 2012a,b, 2013); (iv) flares in active galactic nuclei (AGN) jets (Giannios et al. 2009) or in gamma-ray bursts (Lyutikov 2006a; Lazar et al. 2009); (v) the heating of AGN and microquasar coronae and the observed flares (Di Matteo 1998; Merloni \& Fabian 2001; Goodman \& Uzdensky 2008; Reis \& Miller 2013; Romero et al. 2014; Zdziarski et al. 2014); (vi) the heating of the lobes of giant radio galaxies (Kronberg et al. 2004); (vii) transient outflow production in microquasars and quasars (de Gouveia dal Pino \& Lazarian 2005; de Gouveia Dal Pino et al. 2010; Kowal et al. 2011; McKinney et al. 2012; Dexter et al. 2014); (viii) gamma-ray burst outflows and non-thermal emissions (Drenkhahn \& Spruit 2002; Giannios \& Spruit 2007; McKinney \& Uzdensky 2012); (ix) X-ray flashes (Drenkhahn \& Spruit 2002); or (x) soft gamma-ray repeaters (Lyutikov 2006b; Uzdensky 2011).

In all these cases, it is crucial to know the amount of magnetic energy transferred to the particles during a reconnection event, the relative fraction distributed to ions and electrons, as well as the distribution in momentum space of the accelerated particles. The aim of this paper is to shed light on these questions. In the literature, several acceleration mechanisms by magnetic reconnection have been identified, which we briefly review below. Their relative importance depends on the plasma parameters and on the magnetic field geometry.

One acceleration mechanism occurs when particles are trapped in contracting magnetic islands and thus accelerated by the induced electric field when they are reflected on the two approaching sides. It can be efficient in collisionless plasmas (Drake et al. 2006, 2010; Bessho \& Bhattacharjee 2012) or in collisional plasmas (Kowal et al. 2011) where reconnection is fast because of turbulence. For a closer analysis, the ratio of Larmor radii to typical sizes of the magnetic islands is key. In non-relativistic plasmas, particle Larmor radii are smaller than the magnetic gradient scales, particle motions are adiabatic inside and around the islands, and particle-in-cell (PIC) simulations and analytical estimations agree that this mechanism produces power-law spectra, with indexes $p=1.3$ or softer depending on the plasma $\beta$ and island aspect ratio (Drake et al. 2006). In plasmas with relativistic magnetizations, the Larmor radii of the accelerated particles likely exceed the island scales, at least at early times, so that another analytical approach has to be employed (Bessho \& Bhattacharjee 2012), and there is no analytical expression for the resulting spectra. PIC simulations in relativistic pair plasmas show that this mechanism contributes significantly to the building of the high-energy population (Bessho \& Bhattacharjee 2012; Sironi \& Spitkovsky 2014), a result that we confirm to also hold for relativistic ion-electron reconnection. In addition, since the island edges are the place 
of strong motional electric fields, particles can gain energy there without necessarily turning around the whole island. Liu et al. (2011) report that in their relativistic pair plasma simulations, most particles are energized in this way. Jaroschek et al. (2004) also find that this scenario is important.

Another acceleration mechanism, also relying on the firstorder Fermi process and on stochasticity, is the bouncing motion of particles between the two inflows converging from both sides of the current sheet. Energy is gained when the particle turns around, and is transferred by the motional electric field $\boldsymbol{E}=-\boldsymbol{v} \wedge \boldsymbol{B}$ present in the inflow. Drury (2012) derives the powerlaw spectrum for non-relativistic particles: $\mathrm{d} n(v) / \mathrm{d} v \propto v^{-p}$ with $v$ the velocity, $p=(r+2) /(r+1)$, where $r=n_{\text {out }} / n_{\text {in }}$ is the compression ratio that is not restricted to low values as in the case of shocks. Giannios (2010) derives the maximal Lorentz factor produced in the relativistic case, and Bosch-Ramon (2012) discusses conditions for entering this acceleration regime. This mechanism does not rely on a direct acceleration by the reconnection electric field $E_{\text {rec }}$ when particles are demagnetized at the center of the diffusion region, but makes use of the motional electric field in the inflow. It is thus efficient in non-relativistic and collisional plasmas (Kowal et al. 2011) where direct acceleration by $E_{\mathrm{rec}}$ is known to be negligible. It requires particles crossing the current sheet and bouncing off the other side, i.e., having a Larmor radius in the asymptotic field that is larger than the sheet width, which is generally true only for pre-accelerated particles or hot inflows. We show here that for cold inflows and relativistic setups, electrons and ions do not cross the current sheet, and so do not undergo this acceleration mechanism.

A third acceleration mechanism is by the reconnection electric field $E_{\text {rec }}$, which is initially induced by magnetic field flux variations and sustained in steady or quasi-steady state by the non-ideal response of the plasma. In the diffusion region, the condition $E>B$ for antiparallel reconnection, or $\boldsymbol{E} \cdot \boldsymbol{B} \neq 0$ if there is a guide field, defines an acceleration region where particles can be freely accelerated and directly gain energy. In any case, the reconnection electric field is alone responsible for transfering energy between the magnetic field and the particles, and thus obviously for accelerating particles. But to what extent this kinetic energy is distributed between the bulk flow velocity of the outflows, their thermal energy, and a possible high-energy tail, as well as the properties of the high-energy tail, are open questions. This mechanism is inefficient for non-relativistic reconnection because the acceleration zone is too short (along the outflow direction) (Drake et al. 2010; Kowal et al. 2011; Drury 2012) and affects too few particles, but is efficient under relativistic conditions where the larger reconnection electric field creates a wider acceleration zone (Zenitani \& Hoshino 2001, 2007). It has indeed been found, with PIC simulations of relativistic reconnection, that power-law tails are produced through particle acceleration by $E_{\mathrm{rec}}$. Several indexes are found, for example, measuring the index $p$ as $\mathrm{d} n_{\mathrm{e}} / \mathrm{d} \gamma \propto \gamma^{-p}$ and retaining only relativistic PIC simulations that all concern pair plasmas: Zenitani \& Hoshino (2001) (2D): $p=1$ for particles around the $\mathrm{X}$-point and for the total spectra; Zenitani \& Hoshino (2007) (2D): $p=3.2$ and 2.4 at late times; Jaroschek et al. (2004) (2D): $p=1$ for particles around the X-point, modified to $p=3$ by island acceleration in the whole domain; Jaroschek et al. (2008) (2D, two colliding current sheets): power law; Sironi $\&$ Spitkovsky (2011a) (2D, stripped pulsar wind): $p=1.4$ after the shock; Cerutti et al. (2013) (2D): $p=3.8$; Sironi \& Spitkovsky (2014) (2D without guide field): $p=4,3,2,1.5$ for inflow magnetizations $\sigma=1,3,10,30,50$ and a saturation above 50 , and $p=2.3$ in 3D with $\sigma=10$. On the other hand, Kagan et al. (2013) (3D) find a high-energy tail but interpret it as not having a power-law shape. On the analytical side, Zenitani \& Hoshino (2001) present a toy model predicting power laws, and Bessho \& Bhattacharjee (2012) derive the spectrum of particles escaping from an antiparallel X-point (see Sect. 4.3.3). Also, it is noticeable that the ultrarelativistic test particle simulations of Cerutti et al. (2012a) produce very hard power laws $(p \sim-0.5)$, with electrons accelerated along Speiser orbits without any stochasticity. This diversity of results calls for a unified analysis of simulations with various initial configurations, which we aim to provide here.

Other acceleration mechanisms exist, especially far from the diffusion region. A first example is stochastic acceleration in the turbulence associated with reconnection (Kowal et al. 2011; Hoshino 2012). A second, important example is at the magnetic separatrices that separate the non-reconnected/reconnected regions, where plasma flows through a nonlinear wave structure (see also Sect. 4.3). Particle acceleration should also occur at the dipolarization front. Our simulation setup with no localized initial perturbation precludes the existence of these other mechanisms, and instead we focus on acceleration close to the diffusion region and inside islands, which is likely to be important in relativistic setups.

This manuscript is dedicated to relativistic ion-electron plasmas, for which no study exists yet. These plasmas are probably present in AGN and microquasar coronae, in microquasar jets (Kotani et al. 1994; Díaz et al. 2013), or possibly in GRB and AGN jets. Physical parameters are discussed in Sect. 4.3.

\section{Simulation setups}

\subsection{The simulations}

We performed 2D PIC simulations of magnetic reconnection, mainly in an ion-electron plasma of mass ratio $m_{\mathrm{i}} / m_{\mathrm{e}}=25$. We also present one simulation for each value $m_{\mathrm{i}} / m_{\mathrm{e}}=1,12$, and 50. We used the explicit PIC code Apar-T, presented in Melzani et al. (2013). The simulations are the same as those of Melzani et al. (2014b), to which we refer for details. The initial state is a Harris equilibrium with a reversing magnetic field

$\boldsymbol{B}_{\text {rec }}=\hat{z} B_{0} \tanh (x / L)$,

plus in some cases a guide field $\boldsymbol{B}_{\mathrm{G}}=B_{\mathrm{G}} \hat{\boldsymbol{y}}$. The magnetic field $\boldsymbol{B}_{\text {rec }}$ is produced by a current sheet (abbreviated cs), which is formed by counter-streaming ions and electrons following the density profile $n_{\mathrm{cs}}(x)=n_{\mathrm{cs}}(0) / \cosh ^{2}(x / L)$, with bulk velocities $U_{\mathrm{e}}$ and $U_{\mathrm{i}}=-U_{\mathrm{e}}$ in the $\pm y$ directions. We denote the associated Lorentz factors by $\Gamma_{\mathrm{e}}$ and $\Gamma_{\mathrm{i}}$. Each species follows a Maxwell-Jüttner distribution. The parameters of the current sheet are given in Table 2 . They actually differ by $\sim 5 \%$ from the values of the actual kinetic equilibrium, in order to speed up the otherwise slow initial phase. We note that this initial perturbation is not localized in space, so that islands and X-point form everywhere along the current sheet.

In addition, there is a background plasma at rest, with number density $n_{\mathrm{bg}}$ equal for ions and electrons, and temperatures $T_{\mathrm{bg}, \mathrm{i}}$ and $T_{\mathrm{bg}, \mathrm{e}}$.

The free parameters are the characteristics of the background plasma $\left(n_{\mathrm{bg}} / n_{\mathrm{cs}}(0), T_{\mathrm{bg}, \mathrm{i}}\right.$ and $\left.T_{\mathrm{bg}, \mathrm{e}}\right)$ : the strength of the guide field $B_{\mathrm{G}} / B_{0}$; the width of the magnetic field reversal in electron inertial lengths $L / d_{\mathrm{e}}$; and the magnetization of the current sheet plasma with respect to the asymptotic magnetic field, here expressed via $\omega_{\mathrm{ce}} / \omega_{\mathrm{pe}}\left(\omega_{\mathrm{ce}}=e B_{0} / m_{\mathrm{e}}\right.$ is the electron cyclotron frequency in the asymptotic magnetic field $B_{0}, \omega_{\mathrm{pe}}^{2}=n_{\mathrm{e}} e^{2} /\left(\epsilon_{0} m_{\mathrm{e}}\right)$ 
M. Melzani et al.: The energetics of relativistic magnetic reconnection

Table 1. Parameters of the inflow (or background) plasma, and resulting power-law index $p$, sorted in order of increasing magnetization (except for the last line).

\begin{tabular}{|c|c|c|c|c|c|c|c|c|c|}
\hline$\omega_{\mathrm{ce}} / \omega_{\mathrm{pe}}$ & $n_{\mathrm{bg}} / n_{\mathrm{cs}}(0)$ & $B_{\mathrm{G}} / B_{0}$ & $\sigma_{\mathrm{i}+\mathrm{e}}$ & $V_{\mathrm{A}, \text { in }}^{\mathrm{R}} / c$ & & $T_{\mathrm{bg}, s}(\mathrm{~K})$ & $\beta_{s}$ & $\sigma_{s}^{\text {hot }}\left(B_{\mathrm{rec}}\right)$ & $p=-\operatorname{dlog} n(\gamma) / \operatorname{d} \log \gamma$ \\
\hline $\begin{array}{l}1 \\
\left(m_{\mathrm{i}}=25 m_{\mathrm{e}}\right)\end{array}$ & 0.1 & 0 & 0.38 & 0.53 & $\begin{array}{l}\text { ion } \\
\text { elec. }\end{array}$ & $\begin{array}{c}1.5 \times 10^{7} \\
\prime \prime\end{array}$ & $\begin{array}{c}5 \times 10^{-4} \\
\end{array}$ & $\begin{array}{l}0.4 \\
9.9 \\
\end{array}$ & $\begin{array}{c}\text { no power law } \\
4-5\end{array}$ \\
\hline $\begin{array}{l}3 \\
\left(m_{\mathrm{i}}=25 m_{\mathrm{e}}\right)\end{array}$ & 0.31 & 0 & 1.11 & 0.73 & $\begin{array}{l}\text { ion } \\
\text { elec. }\end{array}$ & $\begin{array}{c}2 \times 10^{8} \\
11\end{array}$ & $\begin{array}{c}2.5 \times 10^{-3} \\
\prime \prime\end{array}$ & $\begin{array}{l}1.2 \\
27 \\
\end{array}$ & $\begin{array}{c}8 \\
2.2-2.6\end{array}$ \\
\hline $\begin{array}{l}3 \\
\left(m_{\mathrm{i}}=25 m_{\mathrm{e}}\right)\end{array}$ & 0.1 & 0 & 3.26 & 0.88 & $\begin{array}{r}\text { ion } \\
\text { elec. }\end{array}$ & $\begin{array}{l}2 \times 10^{8} \\
3 \times 10^{9} \\
\end{array}$ & $\begin{array}{l}7.5 \times 10^{-4} \\
1.1 \times 10^{-2} \\
\end{array}$ & $\begin{array}{l}3.6 \\
35 \\
\end{array}$ & $\begin{array}{l}6.5 \\
2.8 \\
\end{array}$ \\
\hline $\begin{array}{l}3 \\
\left(m_{\mathrm{i}}=25 m_{\mathrm{e}}\right)\end{array}$ & 0.1 & 0 & 3.46 & 0.88 & $\begin{array}{r}\text { ion } \\
\text { elec. }\end{array}$ & $\begin{array}{c}1.5 \times 10^{7} \\
\end{array}$ & $\begin{array}{c}5.6 \times 10^{-5} \\
\end{array}$ & $\begin{array}{l}3.6 \\
89 \\
\end{array}$ & $\begin{array}{l}\text { not investigated } \\
\text { " }\end{array}$ \\
\hline$\left(m_{\mathrm{i}}=25 m_{\mathrm{e}}\right)$ & $\begin{array}{c}0.1 \\
\text { Identical to }\end{array}$ & $\begin{array}{c}0 \\
\text { above, b }\end{array}$ & $\begin{array}{l}3.45 \\
\text { large }\end{array}$ & $\begin{array}{c}0.88 \\
\text { ox }(888\end{array}$ & $\begin{array}{l}\text { ion } \\
\text { elec. } \\
1138\end{array}$ & $\begin{array}{c}2 \times 10^{8} \\
\text { instead of }\end{array}$ & $\begin{array}{c}7.5 \times 10^{-4} \\
\prime \prime \\
55 \times 683 d_{\mathrm{e}}\end{array}$ & $\begin{array}{c}3.6 \\
83 \\
\text { nd duration }\end{array}$ & $\begin{array}{c}5.8 \\
1.5-2 \\
4.8 \\
1.8 \\
\end{array}$ \\
\hline $\begin{array}{l}3 \\
\left(m_{\mathrm{i}}=25 m_{\mathrm{e}}\right)\end{array}$ & 0.1 & 0.5 & 3.46 & 0.81 & $\begin{array}{l}\text { ion } \\
\text { elec. }\end{array}$ & $\underset{\prime \prime}{1.5 \times 10^{7}}$ & $\underbrace{4.5 \times 10^{-5}}_{\prime \prime}$ & $\begin{array}{l}3.6 \\
89 \\
\end{array}$ & $\begin{array}{c}8 \\
\text { no power law }\end{array}$ \\
\hline $\begin{array}{l}3 \\
\left(m_{\mathrm{i}}=25 m_{\mathrm{e}}\right)\end{array}$ & 0.1 & 1 & 3.46 & 0.66 & $\begin{array}{l}\text { ion } \\
\text { elec. }\end{array}$ & $1.5 \times 10^{7}$ & $2.8 \times 10^{-5}$ & $\begin{array}{l}3.6 \\
89\end{array}$ & $\begin{array}{c}8 \\
1.5\end{array}$ \\
\hline $\begin{array}{l}6 \\
\left(m_{\mathrm{i}}=25 m_{\mathrm{e}}\right)\end{array}$ & 0.1 & 0 & 13.5 & 0.97 & $\begin{array}{l}\text { ion } \\
\text { elec. }\end{array}$ & $\begin{array}{c}8 \times 10^{8} \\
\prime \prime\end{array}$ & $\begin{array}{c}7.5 \times 10^{-4} \\
\prime \prime\end{array}$ & $\begin{array}{c}14 \\
260\end{array}$ & $\begin{array}{l}3.6 \\
1.2 \\
\end{array}$ \\
\hline $\begin{array}{l}3 \\
\text { (pairs) }\end{array}$ & 0.1 & 0 & 41.4 & 0.988 & $\begin{array}{c}\text { ion } \\
\text { elec. }\end{array}$ & $\begin{array}{c}2 \times 10^{8} \\
\end{array}$ & $\begin{array}{c}7.5 \times 10^{-4} \\
\prime \prime\end{array}$ & $\begin{array}{l}83 \\
\prime \prime\end{array}$ & $\begin{array}{l}1.5 \\
\prime \prime\end{array}$ \\
\hline $\begin{array}{l}3 \\
\left(m_{\mathrm{i}}=12 m_{\mathrm{e}}\right)\end{array}$ & 0.1 & 0 & 6.9 & 0.93 & $\begin{array}{l}\text { ion } \\
\text { elec. }\end{array}$ & $2 \times 10^{8}$ & $\underset{1}{7.5 \times 10^{-4}}$ & $\begin{array}{l}7.5 \\
83\end{array}$ & $\begin{array}{l}3.6 \\
1.5\end{array}$ \\
\hline $\begin{array}{l}6 \\
\left(m_{\mathrm{i}}=50 m_{\mathrm{e}}\right)\end{array}$ & 0.1 & 0 & 6.9 & 0.93 & $\begin{array}{r}\text { ion } \\
\text { elec. }\end{array}$ & $\begin{array}{c}8 \times 10^{8} \\
1\end{array}$ & $\begin{array}{c}7.5 \times 10^{-4} \\
\end{array}$ & $\begin{array}{l}7.1 \\
260\end{array}$ & $\begin{array}{c}5 \\
1.5 \\
\end{array}$ \\
\hline
\end{tabular}

Notes. The background plasma $\beta_{s}=n_{s} T_{s} /\left(B^{2} / 2 \mu_{0}\right)=2 \Theta_{s} / \sigma_{s}^{\text {cold }}(B)$ includes the guide field. The magnetization $\sigma_{s}^{\text {hot }}$ is defined by Eq. (2), and $\sigma_{\mathrm{i}+\mathrm{e}}$ is the total magnetization (Eq. (5)). The Alfvén speed $V_{\mathrm{A}, \mathrm{in}}^{\mathrm{R}}$ is defined by Eq. (4). When there is a guide field, the value displayed is $V_{\mathrm{A}, \mathrm{R}}^{\mathrm{R}} \cos \theta$ (Sect. 2.1). The index of the power-law component of the background population (when there is one) is $p$.

is the non-relativistic electron plasma frequency at the current sheet center at $t=0$, and $\left.d_{\mathrm{e}}=c / \omega_{\mathrm{pe}}\right)$. The background plasma magnetization results from the above variables. The simulations and the background plasma parameters and magnetizations are listed in Table 1.

We now give the definition of the magnetizations. The inflow or background plasma magnetization for species $s$ is defined as the ratio of the energy flux in the reconnecting magnetic field to the energy flux in the inflowing particles (Melzani et al. 2014b):

$$
\begin{aligned}
\sigma_{s}^{\mathrm{hot}}\left(B_{\mathrm{rec}}\right) & =\frac{E \times B_{\mathrm{rec}} / \mu_{0}}{n_{\mathrm{lab}, s}\left\langle v \gamma m_{s} c^{2}\right\rangle_{s}}=\frac{B_{\mathrm{rec}}^{2} / \mu_{0}}{n_{\mathrm{lab}, s} m_{s} c^{2} \Gamma_{s} h_{0, s}} \\
& =\frac{\sigma_{s}^{\mathrm{cold}}\left(B_{\mathrm{rec}}\right)}{\Gamma_{s} h_{0, s}} .
\end{aligned}
$$

Here we used $E=v_{\text {in, } s} B$ in the ideal inflowing plasma, and the relation $\langle v \gamma\rangle_{s}=h_{0, s} \Gamma_{s} v_{\text {in,s }}$ (Melzani et al. 2013), where $\langle\cdot\rangle_{s}$ denotes an average over the momentum distribution function, with $h_{0, s}$ the comobile enthalpy, and $\Gamma_{s}=\left(1-v_{\mathrm{in}, s}^{2} / c^{2}\right)^{-1 / 2}$. Also, $n_{\mathrm{lab}, s}$ is the lab-frame particle number density, and $\sigma_{s}^{\text {cold }}$ the magnetization of the plasma without taking temperature effects or relativistic bulk motion into account:

$\sigma_{s}^{\text {cold }}(B)=\frac{B^{2}}{\mu_{0} n_{\mathrm{lab}, s} m_{s} c^{2}}$

Finally, the total magnetization of the plasma is

$\sigma_{\mathrm{i}+\mathrm{e}}\left(B_{\mathrm{rec}}\right)=\frac{B_{\mathrm{rec}}^{2} / \mu_{0}}{\sum_{s} n_{\mathrm{lab}, s} m_{s} c^{2} \Gamma_{s} h_{0, s}}=\frac{\sigma_{\mathrm{i}}^{\text {cold }}\left(B_{\mathrm{rec}}\right)}{\sum_{s} \Gamma_{s}\left(m_{s} / m_{\mathrm{i}}\right) h_{0, s}}$.
Table 2. Parameters of the current sheet.

\begin{tabular}{lcccccc}
\hline \hline$\frac{m_{\mathrm{i}}}{m_{\mathrm{e}}}$ & $\omega_{\mathrm{ce}} / \omega_{\mathrm{pe}}$ & $L / d_{\mathrm{i}}$ & $\Gamma_{\mathrm{e}} \beta_{\mathrm{e}}$ & $\Theta_{\mathrm{e}}$ & $\Theta_{\mathrm{i}}$ & $L / r_{\mathrm{ce}}$ \\
\hline 1 & 3 & 2.5 & 0.53 & 2.40 & 2.40 & 1.6 \\
12 & 3 & 0.5 & 0.53 & 2.40 & 0.2 & 1.1 \\
25 & 1 & 0.5 & 0.20 & 0.25 & $1.0 \times 10^{-2}$ & 3.8 \\
25 & 3 & 0.5 & 0.53 & 2.40 & $9.6 \times 10^{-2}$ & 1.6 \\
25 & 6 & 1 & 0.70 & 10 & 0.4 & 1.5 \\
50 & 6 & 0.7 & 0.60 & 10 & 0.2 & 1.5 \\
\hline
\end{tabular}

Notes. To each row in the table different background plasma parameters can correspond, hence different simulations. The full simulation list is presented in Table 1. Here, the electron and ion temperatures are the same, denoted by $\Theta_{\mathrm{e}}=T_{\mathrm{e}} /\left(m_{\mathrm{e}} c^{2}\right)$ and $\Theta_{\mathrm{i}}=T_{\mathrm{i}} /\left(m_{\mathrm{i}} c^{2}\right)$. The electrons and ions counterstream with opposite velocities $\pm \beta_{\mathrm{e}} c$ and associated Lorentz factors $\Gamma_{\mathrm{e}}$. The sheet half-width in units of ion inertial lengths is $L / d_{\mathrm{i}}$, while in units of electron thermal Larmor radii it is $L / r_{\text {ce }}$.

For non-relativistic temperatures $\left(h_{0, s} \sim 1\right)$ and non-relativistic inflow velocities $\left(\Gamma_{s} \sim 1\right)$, the total magnetization reduces to $\sigma_{\mathrm{i}+\mathrm{e}} \simeq \sigma_{\mathrm{i}}^{\text {cold }} /\left(1+m_{\mathrm{e}} / m_{\mathrm{i}}\right) \simeq \sigma_{\mathrm{e}}^{\text {cold }} /\left(1+m_{\mathrm{i}} / m_{\mathrm{e}}\right)$.

The relativistic Alfvén velocity in the inflow is defined by

$\left(\frac{V_{\mathrm{A}, \mathrm{in}}^{\mathrm{R}}}{c}\right)^{2}=\frac{\sigma_{\mathrm{i}+\mathrm{e}}\left(B_{\mathrm{tot}}\right)}{1+\sigma_{\mathrm{i}+\mathrm{e}}\left(B_{\mathrm{tot}}\right)}$,

with $B_{\text {tot }}=\left(B_{0}^{2}+B_{\mathrm{G}}^{2}\right)^{1 / 2}$, and $\sigma_{\mathrm{i}+\mathrm{e}}\left(B_{\mathrm{tot}}\right)$ the comobile plasma total magnetization (Eq. (4) with $\Gamma_{s}=1$ and $n_{\mathrm{lab}, s}$ replaced by the comobile number density). When there is a guide field, it is relevant to project the Alfvén velocity 5 in the plane of the reconnecting magnetic field: $V_{\mathrm{A}, \text { in }}^{\mathrm{R}} \cos \theta$ with $\tan \theta=B_{\mathrm{G}} / B_{0}$. This 
is needed to correctly normalize the reconnection electric field (Melzani et al. 2014b).

\subsection{Resolution and domain size}

The numerical resolution is set by the number of cells $n_{x}$ per electron inertial length $d_{\mathrm{e}}=c / \omega_{\mathrm{pe}}$, by the number of timesteps $n_{t}$ per electron plasma period $2 \pi / \omega_{\text {pe }}$, and by the number of computer particles (the so-called superparticles) per cell $\rho_{\mathrm{sp}}$. The quantities $d_{\mathrm{e}}, \omega_{\mathrm{pe}}$, and $\rho_{\mathrm{sp}}$ are defined at $t=0$ at the center of the current sheet. Here we take $n_{x}=9$ and $n_{t}=150$ (except for $\omega_{\mathrm{pe}} / \omega_{\mathrm{ce}}=6$ where $\left.n_{t}=250\right)$. We checked by doubling $n_{x}$ and $n_{t}$ that the particle distributions, the energy distribution particle mixing, or the maximal Lorentz factors are not affected by the resolution.

Concerning the number of superparticles per cell at the center of the current sheet, we use $\rho_{\mathrm{sp}}=1090$ for $n_{\mathrm{bg}} / n_{\mathrm{cs}}(0)=0.3$ and $\rho_{\mathrm{sp}}=1820$ for $n_{\mathrm{bg}} / n_{\mathrm{cs}}(0)=0.1$, except for $m_{\mathrm{i}} / m_{\mathrm{e}}=50$ where $\rho_{\mathrm{sp}}=910$. This corresponds, for the case $n_{\mathrm{bg}} / n_{\mathrm{cs}}(0)=0.1$, to 1650 electron and ion superparticles per cell for the plasma of the current sheet, and to 170 for the background plasma. We stress in Melzani et al. (2013, 2014a) that because of their low numbers of superparticles per cell when compared to real plasmas, PIC simulations present high levels of correlations and collisionality, and thus thermalize faster. In the same line of thought, Kato (2013) and May et al. (2014) show that because of these enhanced correlations, high-energy particles are slowed down quickly in PIC plasmas. One should thus ensure that collisionless kinetic processes remain faster than collisional effects, essentially by taking a large enough number $\Lambda^{\mathrm{PIC}}$ of superparticles per Debye sphere and per inertial length sphere, the former constraint being more restrictive. For example, with $\Theta_{\mathrm{e}}=2.4=1.4 \times 10^{10} \mathrm{~K} /\left(m_{\mathrm{e}} c^{2}\right)$, the electron Debye length is 20 cells large, and initially at the center of the current sheet we have $\Lambda^{\text {PIC }} \sim 1820 \times 20 \times 20=7.3 \times 10^{5}$ superparticles. For a background plasma with $T_{\text {bg }}=2 \times 10^{8} \mathrm{~K}$, we have $\Lambda^{\mathrm{PIC}}=133$. We performed a simulation with half as many superparticles per cell, and saw no difference, especially concerning particle distributions, energy repartition, particle mixing, or maximal Lorentz factor. It indicates that we are not affected by $\rho_{\mathrm{sp}}$.

Boundaries are periodic along $z$ and $y$ and reflective along $x$. The simulation with $m_{\mathrm{i}} / m_{\mathrm{e}}=50$ uses a domain size of $8000 \times$ 10240 cells. The number of cells of the simulations with other mass ratios is $4100 \times 6144$, corresponding to $455 \times 683$ initial electron inertial lengths $d_{\mathrm{e}}$, typically with $4 \times 10^{9}$ superparticles. We performed a simulation with twice as small a domain along $z$ : particle distributions are identical as long as there is a significant number of islands and X-points in the domain $(\geq 4)$, but differ afterward. In the smaller simulation, the distribution cutoff is at lower energies, and the power laws are steeper (softer). We also performed a simulation with a larger domain $\left(8000 \times 10240\right.$ cells, i.e., $888 \times 1138$ inertial lengths $\left.d_{\mathrm{e}}\right)$ and otherwise identical parameters: the electron distribution saturates identically to the $4100 \times 6144$ case, but the ion distribution reaches a harder final state. It indicates that our domain size and simulation duration are large enough for electrons, but possibly not for ions. The latter may build harder spectra and reach higher energies in real systems.

\subsection{Diagnostics}

We initially select roughly 200000 particles (out of the 4 to 14 billion in total) uniformly in space and write their positions, velocities, as well as the magnetic and electric fields they undergo, once every few timesteps. The visualization of these data is performed with the visualization software VisIt (Childs et al. 2012). We divide the followed particles into two populations: those that are initially in the current sheet (colored in red), and those initially outside (marked in green). In other words, red particles are those satisfying

distance from middle plane at $t=0<2 L$,

while green particles satisfy

distance from middle plane at $t=0>2 L$.

Changing the limiting length from $2 L$ to between $1.5 L$ and $3 L$ has been confirmed as not influencing the presented results. An example is shown in Fig. 1. As we show, these two populations almost do not mix spatially, and they undergo very different acceleration mechanisms, resulting in completely different particle energy distributions. Particles from the background plasma accelerated by the reconnection are expected to dominate in number and energy for very large systems. This is why we focus more on the green population.

\section{Results}

We first present results from a reference simulation in Sect. 3.1, and then study modifications due to varying the background particle number density, magnetic field, or temperature without considering a guide field in Sect. 3.2. The consequences of a guide field are explored in Sect. 3.3.

\subsection{Main case}

We start by presenting the results of the simulation with $\omega_{\mathrm{ce}} / \omega_{\mathrm{pe}}=3, n_{\mathrm{bg}}=0.1 n_{\mathrm{cs}}(0), T_{\mathrm{bg}, \mathrm{i}}=T_{\mathrm{bg}, \mathrm{e}}=2 \times 10^{8} \mathrm{~K}$, resulting in a magnetization in the background plasma $\sigma_{\mathrm{i}, \mathrm{e}}^{\text {hot }}=3.6,83$ for ions and electrons, respectively (defined by Eq. (2), see also Tables 1 and 2) and in an inflow Alfvén speed $V_{\mathrm{A} \text {,in }}^{\mathrm{R}}=0.88 c$.

The background electron distribution (Fig. 1) starts rising and taking a power-law shape around $t=500 \omega_{\mathrm{ce}}^{-1}$, which corresponds to the starting of the reconnection instability. What happens is that the reconnection electric field $E_{\text {rec }}$ spreads in the background plasma and sets the particles into motion in an $E \times B$ drift directed toward the current sheet. More and more background particles thus pass in the current sheet, where they are demagnetized and able to gain energy via $E_{\text {rec }}$. The power-law component thus comprises more and more particles. After gaining energy in the acceleration zone, particles accumulate around the magnetic islands and swirl around them. They are subsequently accelerated when islands merge and contract. The power-law index passes from a soft initial value of $p=-\mathrm{d} \log n(\gamma) / \mathrm{d} \log \gamma=3$ to a harder final value that converges to $p \sim 1.8$. We stopped the simulation at $t=3750 \omega_{\mathrm{ce}}^{-1}$, when there were still enough islands and $\mathrm{X}$-points so that we are not affected by boundaries (Sect. 2.2).

The distribution of the current sheet electrons is shown at $t=0$ by a red dashed line in Fig. 1, and consists then in a thermal hot plasma. When the reconnection instability starts, this population is heated by the formation and contraction of islands. This heating slowly progresses at later times as islands merge, to result in the solid red curve of Fig. 1.

The background magnetization of ions is only slightly relativistic $\left(\sigma_{i}^{\text {hot }}=3.6\right)$. The current sheet population is heated, while the background population distribution is power-law like, 
$\omega_{\mathrm{ce}} / \omega_{\mathrm{pe}}=3, n_{\mathrm{bg}}=0.1 n_{\mathrm{cs}}(0), T_{\mathrm{bg}, \mathrm{e}}=T_{\mathrm{bg}, \mathrm{i}}=2 \times 10^{8} \mathrm{~K}$
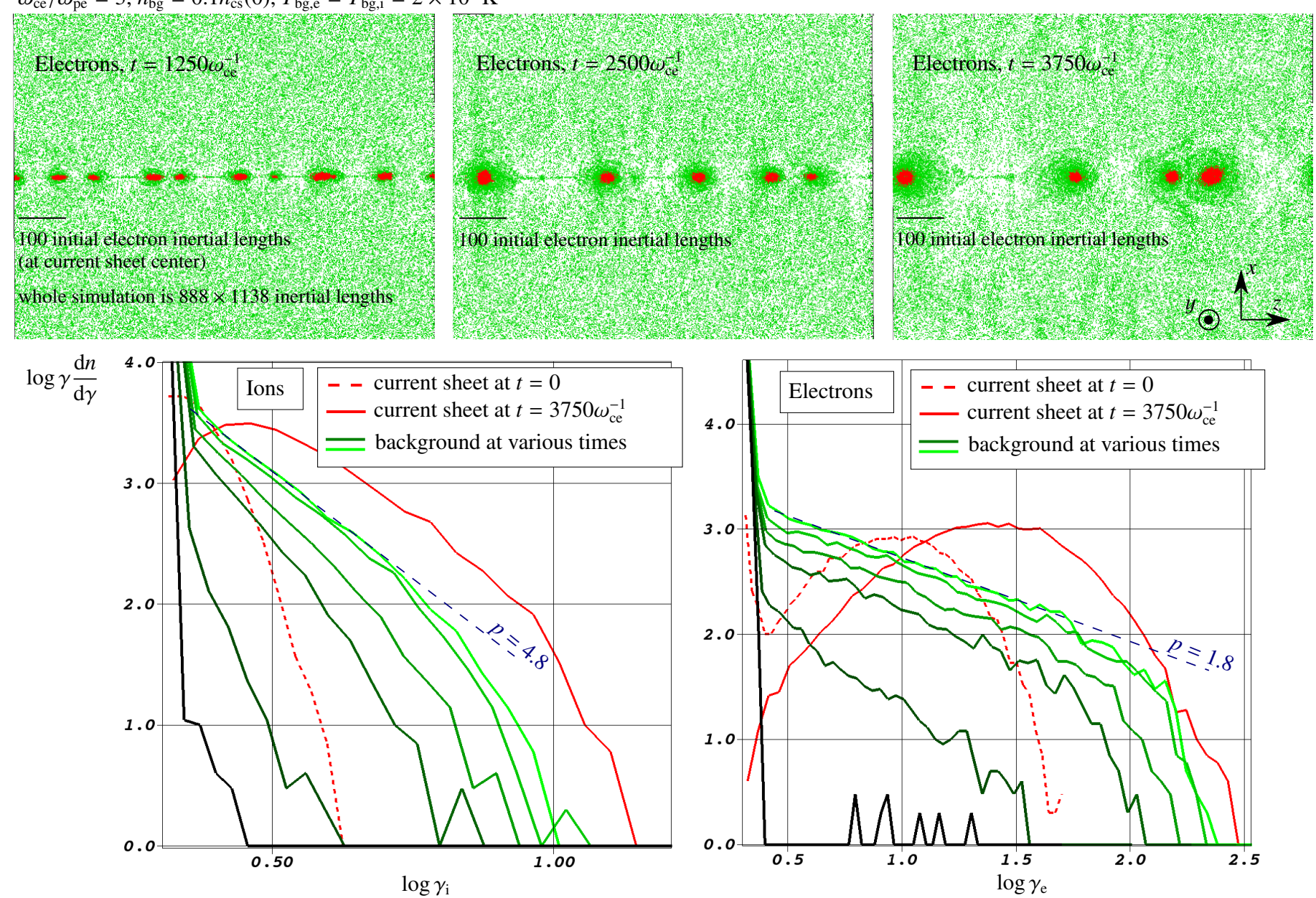

Fig. 1. Data from the main simulation (Sect. 3.1), with a background magnetization respectively for ions and electrons $\sigma_{\mathrm{i}, \mathrm{e}}^{\text {hot }}=3.6,83$. Top: snapshots of a random selection of electrons in the whole simulation domain. Red particles are inside the current sheet at $t=0$, green ones are outside. Bottom: Lorentz factor distributions. Red (green) curves concern the red (green) population. For the green curves, times are ordered as dark to light green, with values $0,750,1500,2250,3000,3750 \omega_{\mathrm{ce}}^{-1}$, i.e., one curve every $750 \omega_{\mathrm{ce}}^{-1}=250 \omega_{\mathrm{pe}}^{-1}=50 \omega_{\mathrm{pi}}^{-1}=30 \omega_{\mathrm{ci}}^{-1}$. The blue dashed line indicates the final power-law slope of the background accelerated particles.

with a final index $p \sim 4.8$. This is similar to non-relativistic simulations where all species form steep spectra.

It is evident from the top panels of Fig. 1 that the green electrons from the background plasma do not penetrate deep inside the islands and, on the other hand, that the red electrons initially from the current sheet do not escape from the islands, even when they merge. The two populations thus remain almost separated. This is because particles from the background plasma are scattered by the strong magnetic field structure surrounding the islands and thus swirl around these field lines, performing circles around the islands but not reaching the inside. On their side, red particles from the islands cannot escape because of the very same magnetic field structure. This remains true for ions, but less so because of their larger Larmor radius. Figure 5 illustrates this population separation for several simulations.

The energy distribution between fields and particles is shown in Fig. 2 (top). This energy is computed over a fixed rectangle in space, defined to include all particles that will reach the center of the current sheet before the end of the simulation. It thus excludes regions that, because of the finite simulation length, are never in contact with the current sheet. Energies are normalized by $\mathcal{E}_{0}$, the total initial energy in this area, which is to $\sim 90 \%$ the energy in the magnetic field. The energy in the magnetic field is transferred to the kinetic energy of the particles $\left(0.6 \mathcal{E}_{0}\right.$ in the final state), to the reconnected magnetic field $B_{x}\left(0.2 \mathcal{E}_{0}\right)$, and to the reconnection electric field $E_{y}\left(0.03 \mathcal{E}_{0}\right)$. The components $B_{y}$, $E_{x}$, and $E_{z}$ get a far smaller amount of energy $\left(\sim 0.005 \mathcal{E}_{0}\right)$. A first conclusion is that the portion of dissipated magnetic energy is large. Table 3 presents this analysis for several simulations. It shows that the amount of dissipated magnetic energy is even larger at larger inflow magnetization. A second important aspect is the energy distribution between ions and electrons. In Fig. 2 (bottom), we show this distribution for the background particles that have been accelerated, i.e., for the particles of the tails in Fig. 1. The ions weight as $60 \%$ of this kinetic energy, the electrons $40 \%$, and this ratio remains constant with time. The same distribution roughly holds for particles in the current sheet (red population). Table 3 shows that this distribution holds for various simulations with different magnetizations and mass ratios, provided that there is no guide magnetic field. We note that the percentages given in Table 3 are not sensitive to the specific time when they are determined. We obtain essentially the same percentages if we repeat the analysis but consider only those particles (and their associated rectangular region and energy $\mathcal{E}_{0}$ ) that reach the current sheet before half of the total simulation time. 

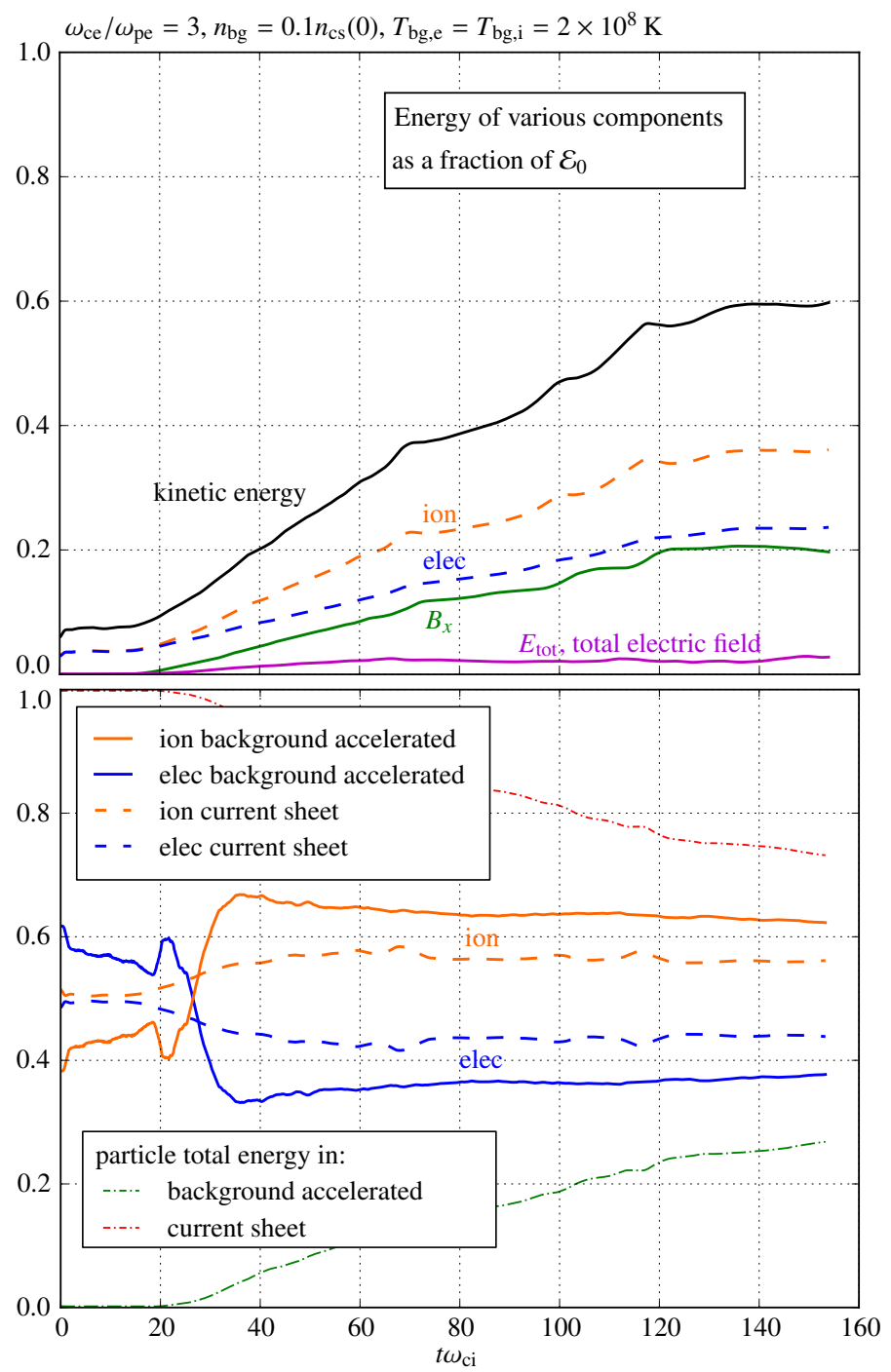

Fig. 2. Energy distribution for the main simulation (Sect. 3.1), with a background magnetization for ions and electrons $\sigma_{\mathrm{i}, \mathrm{e}}^{\text {hot }}=3.6,83$, respectively. Top: energy in the total electric field $E_{\text {tot }}$, in the $x$ component of the magnetic field, and in the particles (also decomposed into the ion and electron contributions). These energies are computed on a fixed area that corresponds to the location of the background particles, or field lines, that reach the current sheet before the end of the simulation. The total initial energy in this area is $\mathcal{E}_{0}$. We note that the energy in $E_{\text {tot }}$ is mostly the energy in the $y$ component $E_{y}$. Also, the energy of $B_{y}$ is $0.5 \%$ of the energy of $B_{x}$. Bottom: orange and blue curves are the energy distribution between ions and electrons for the current sheet particles (dashed), and for the background particles that have been accelerated (solid). They are normalized so that their sum is 1 . The red and green curve show the energy repartition between current sheet particles and background accelerated particles.

\subsection{Case studies, no guide field}

\subsubsection{Influence of the background plasma density}

We now compare the main case with a simulation with identical parameters except for the background plasma number density: $n_{\mathrm{bg}}=0.3 n_{\mathrm{cs}}(0)$ instead of 0.1 , resulting in a smaller magnetization $\sigma_{i, e}^{\text {hot }}=1.2,27$ in the background. The corresponding inflow Alfvén speed is $V_{\mathrm{A} \text {,in }}^{\mathrm{R}}=0.73 c$. The evolution is similar: weak mixing of the two populations, heating of the particles from the current sheet, and acceleration of the particles from the background to form a power law. The power law for electrons has a final index $p$ between 2.2 and 2.6 (Fig. 3), and for ions around $p \sim 8$. This is softer than in the $n_{\mathrm{bg}}=0.1 n_{\mathrm{cs}}(0)$ case, which is expected because a higher background plasma density implies lower magnetization, and as we show here (see also Sironi \& Spitkovsky 2014), softer power laws. It is, however, noticeable that more magnetic energy is transferred to the particles: the kinetic energy is $74 \%$ of the total energy, while it is only $62 \%$ for the case with $n_{\mathrm{bg}}=0.1 n_{\mathrm{cs}}(0)$.

\subsubsection{Influence of the inflow magnetization}

We vary the asymptotic magnetic field strength by varying the parameter $\omega_{\text {ce }} / \omega_{\text {pe }}$ of the Harris equilibrium. Increasing this parameter results in a higher magnetic field and in a hotter current sheet plasma in order to maintain the pressure balance. It allows probing different background plasma magnetizations. Our results indicate harder power laws at higher magnetizations (Fig. 3 for the simulations with $\omega_{\text {ce }} / \omega_{\text {pe }}=1,6$, to also be compared with the simulation $\left.\omega_{\mathrm{ce}} / \omega_{\mathrm{pe}}=3, T_{\mathrm{bg}, \mathrm{e}}=2 \times 10^{8} \mathrm{~K}\right)$. The comparison is as follows:

- The case at low inflow magnetization $\left(\omega_{\mathrm{ce}} / \omega_{\mathrm{pe}}=1, \sigma_{\mathrm{i}, \mathrm{e}}^{\text {hot }}=\right.$ $0.4,9.9, V_{\mathrm{A}, \mathrm{R}}^{\mathrm{R}}=0.53 \mathrm{c}$ ) presents a power-law-like spectrum for background-accelerated electrons (green population) with a large index between 3 and 4, thus decreasing fast and reaching $\gamma_{\max } \sim 10$. The ion distribution is not powerlaw like and is very steep. The final kinetic energy is $48 \%$ of the total initial energy.

- The intermediate case $\left(\omega_{\mathrm{ce}} / \omega_{\mathrm{pe}}=3, \sigma_{\mathrm{i}, \mathrm{e}}^{\mathrm{hot}}=3.6,90, V_{\mathrm{A}, \mathrm{R}}^{\mathrm{R}}=\right.$ $0.88 c$ ) presents a power-law-like spectrum for the background particles with $p \sim 1.5-2$ and $\gamma_{\max } \sim 100$ for electrons, $p \sim 5.8$ for ions. The final kinetic energy is $62 \%$ of the total initial energy.

- The most magnetized and relativistic case $\left(\omega_{\mathrm{ce}} / \omega_{\mathrm{pe}}=6\right.$, $\sigma_{\mathrm{i}, \mathrm{e}}^{\text {hot }}=14,260, V_{\mathrm{A}, \mathrm{R}}^{\mathrm{R}}=0.97 c$ ) exhibits a very flat spectrum for background accelerated electrons, with an index around 1.2, and reaches $\gamma_{\max } \sim 300$. Background accelerated ions have a power-law distribution with index $p \sim 3.6$, which is interestingly close to the index for electrons at the same magnetization (the simulation with $\omega_{\text {ce }} / \omega_{\text {pe }}=1$, where $\sigma_{\mathrm{e}}^{\text {hot }}=9.9$ and $p \sim 4.5$ ), and highlights the relevance of $\sigma_{s}$ of each species to characterize the power law. The final kinetic energy is $73 \%$ of the total initial energy.

For both electrons and ions, this emphasizes that only relativistic reconnection setups (i.e., $\sigma_{s}^{\text {hot }}>1$ for each species $s$ ) can produce power laws, with harder indexes for higher magnetizations.

\subsubsection{Influence of the inflow temperature}

Coming back to the main simulation with $\omega_{\text {ce }} / \omega_{\text {pe }}=3, n_{\text {bg }}=$ $0.1 n_{\mathrm{cs}}(0), T_{\mathrm{bg}, \mathrm{e}}=T_{\mathrm{bg}, \mathrm{i}}=2 \times 10^{8} \mathrm{~K}$, we now increase the background temperature of the electrons to reach $T_{\mathrm{bg}, \mathrm{e}}=3 \times 10^{9} \mathrm{~K}$ (giving $\sigma_{\mathrm{i}, \mathrm{e}}^{\text {hot }}=3.6,35, V_{\mathrm{A}, \text { in }}^{\mathrm{R}}=0.88 c$ ), which is almost the temperature of the current sheet electrons $\left(\Theta_{\mathrm{e}}=2.4=1.4 \times\right.$ $\left.10^{10} \mathrm{~K} /\left(m_{\mathrm{e}} c^{2}\right)\right)$. Electrons from the background plasma already have high energy when reaching the acceleration region, and their initial energy is then a significant fraction of the energy gain furnished by $E_{\mathrm{rec}}$. As a consequence, the power law is less visible (Fig. 3, gray curve). However, as time goes by and as more and more particles from the hot background are accelerated, we expect it to dominate the particle distribution more and more. Its index is $p=2.8$, softer than the colder case. This is 
M. Melzani et al.: The energetics of relativistic magnetic reconnection

Table 3. Energy distribution between fields and particles at the end of different simulations.

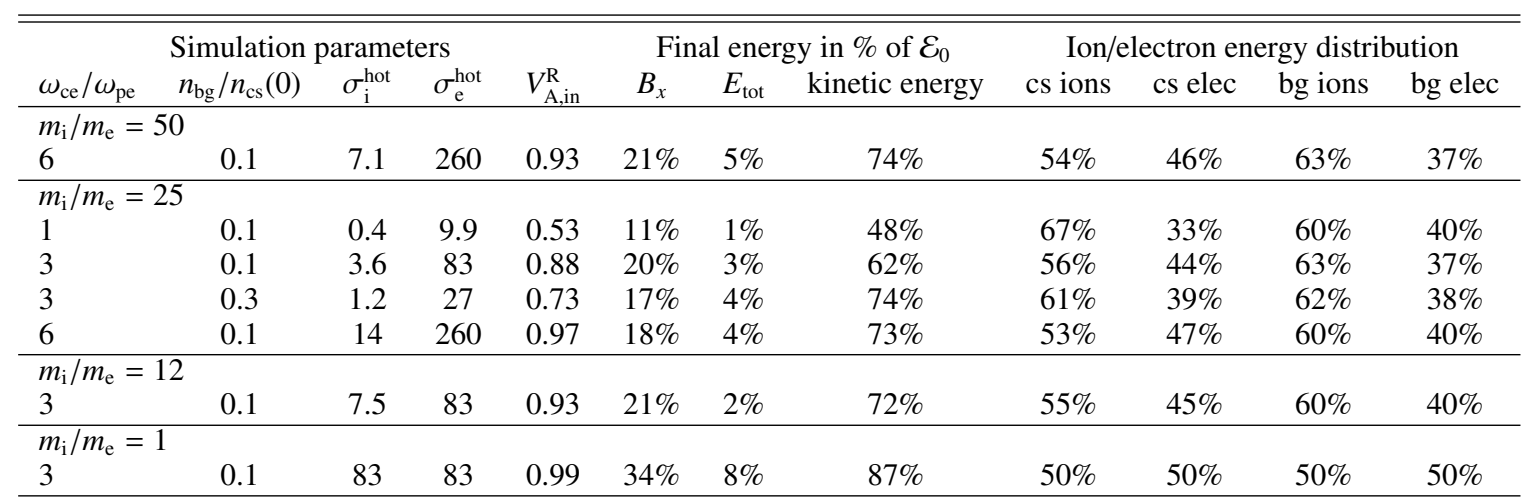

Notes. cs stands for the current sheet population, bg for the background population. The final energy in the electric field is $E_{\text {tot }}$, and is dominated by the energy in $E_{y}$. The energy in $B_{x}$ is denoted by $B_{x}$. As explained in Fig. $2, \mathcal{E}_{0}$ is the total (mostly magnetic) initial energy in the "reconnection area", which is the area from where the particles reach the current sheet before the end of the simulation. Because of the difficulty of precisely measuring this area, the percentage has to be taken with a $\pm 5 \%$ relative uncertainty. The energy distribution between ions and electrons is not affected by this.
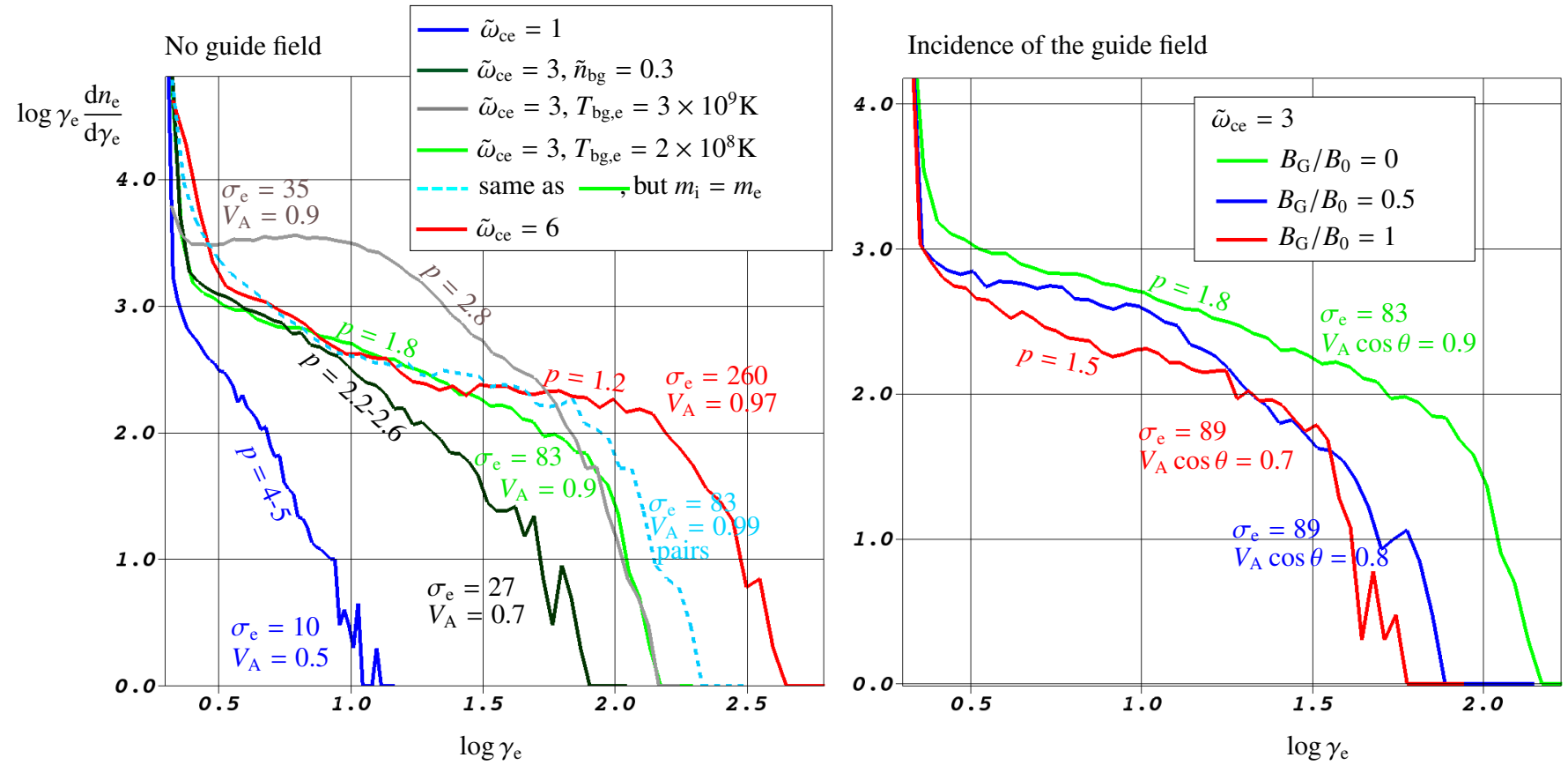

Fig. 3. Lorentz factor distributions for background electrons (green population), for various simulations with $m_{\mathrm{i}} / m_{\mathrm{e}}=25$ or 1 , in the final state. Times are $t=600 \omega_{\mathrm{pe}}^{-1}=1800 \omega_{\mathrm{ce}}^{-1}$ for all simulations, except for $\omega_{\mathrm{ce}} / \omega_{\mathrm{pe}}=1\left(t=1350 \omega_{\mathrm{pe}}^{-1}=1350 \omega_{\mathrm{ce}}^{-1}\right)$, and for $\omega_{\mathrm{ce}} / \omega_{\mathrm{pe}}=6\left(t=600 \omega_{\mathrm{pe}}^{-1}=\right.$ $\left.3600 \omega_{\mathrm{ce}}^{-1}\right)$. Notations are abbreviated: $\tilde{\omega}_{\mathrm{ce}}=\omega_{\mathrm{ce}} / \omega_{\mathrm{pe}}, \tilde{n}_{\mathrm{bg}}=n_{\mathrm{bg}} / n_{\mathrm{cs}}(0), \sigma_{\mathrm{e}}$ stands for $\sigma_{\mathrm{e}}^{\text {hot }}\left(B_{\mathrm{rec}}\right)$, and $V_{\mathrm{A}}$ for $V_{\mathrm{A}, \text { in }}^{\mathrm{R}} \cos \theta / c\left(\right.$ with $\left.\theta=\arctan B_{\mathrm{G}} / B_{0}\right)$. Unless specified, $\tilde{n}_{\mathrm{bg}}=0.1$. The power-law indexes are $p$. The precise setups are reported in Table 1 for each $\sigma_{\mathrm{e}}$. The light-green curve $\left(\sigma_{\mathrm{e}}=83\right.$, $\left.V_{\mathrm{A}}=0.9\right)$ is the same on the left and right panel, and is the final state of the simulation of Fig. 1.

expected because a relativistic temperature decreases the plasma magnetization from 89 to 35 here, and we do have an index close to the one for $n_{\mathrm{bg}}=0.3 n_{\mathrm{cs}}(0)$, which had a similar magnetization $\left(\sigma_{\mathrm{e}}^{\text {hot }}=27, p \sim 2.5\right)$.

Background accelerated ions have a power-law distribution with index $p \sim 6.5$. This is close to their index in the simulation with $\omega_{\text {ce }} / \omega_{\mathrm{pe}}=3, T_{\mathrm{bg}, \mathrm{e}}=T_{\mathrm{bg}, \mathrm{i}}=2 \times 10^{8} \mathrm{~K}$, which is identical except for the initial electron temperature $(p \sim 5.8)$. It shows that electrons have a weak influence on ions.

\subsection{Case studies, guide field}

We now analyze simulations with a guide magnetic field $\boldsymbol{B}_{\mathrm{G}}=$ $B_{\mathrm{G}} \hat{\boldsymbol{y}}$. Figure 3 (right) presents the final electron distributions from simulations with $B_{\mathrm{G}}=0,0.5 B_{0}$, and $B_{0}$. In the intermediate case $\left(B_{\mathrm{G}}=0.5 B_{0}\right)$, the spectrum of the background accelerated electrons shows no clear power law, but extends over a range similar to the no-guide field case. In the strong guide field case $\left(B_{\mathrm{G}}=B_{0}\right)$, the spectrum of the same electron population seems flatter, with a power-law index of $\sim 1.5$ (over a narrow range). The background plasma magnetization in the three simulations is similar. (The guide field is not included in this magnetization parameter, and should not be, because it is not converted into particle energy.) The harder particle distribution should thus be explained by other means. We recall that quite generally, the particles accelerated at the X-point are slowly deflected toward the reconnection exhausts by the $x$ component of $B$. But $B_{x}$ vanishes at the X-point and increases when going 
Table 4. Same as Table 3, but for when there is a guide field. Here $m_{\mathrm{i}} / m_{\mathrm{e}}=25$.

\begin{tabular}{lccccccccccc}
\hline \hline & \multicolumn{3}{c}{ Simulation parameters } & \multicolumn{4}{c}{ Final energy in \% of $\mathcal{E}_{0}$} & \multicolumn{3}{c}{ Ion/electron energy repartition } \\
$\omega_{\mathrm{ce}} / \omega_{\mathrm{pe}}$ & $n_{\mathrm{bg}} / n_{\mathrm{cs}}(0)$ & $\sigma_{\mathrm{i}}^{\text {hot }}$ & $\sigma_{\mathrm{e}}^{\mathrm{hot}}$ & $V_{\mathrm{A}, \mathrm{in}}^{\mathrm{R}}$ & $B_{x}$ & $E_{\mathrm{tot}}$ & kinetic energy & cs ions & cs elec & bg ions & bg elec \\
\hline $\begin{array}{l}B_{\mathrm{G}} / B_{0}=0.5 \\
3\end{array}$ & 0.1 & 3.6 & 89 & 0.81 & $16 \%$ & $4 \%$ & $43 \%$ & $60 \%$ & $40 \%$ & $46 \%$ & $54 \%$ \\
\hline $\begin{array}{l}B_{\mathrm{G}} / B_{0}=1 \\
3\end{array}$ & 0.1 & 3.6 & 89 & 0.66 & $18 \%$ & $5 \%$ & $45 \%$ & $57 \%$ & $43 \%$ & $33 \%$ & $67 \%$ \\
\hline
\end{tabular}

Notes. In this case $\mathcal{E}_{0}$ does not include the guide magnetic field $B_{y}$, because this component is not transferred to the particles (the energy in $B_{y}$ remains constant to within 5\% throughout the simulation).

away from it, so that the farther from the X-point a particle enters the diffusion region, the faster it will be deflected and the less it will be accelerated. The area where an efficient acceleration occurs is thus limited along $z$ by the increase in $B_{x}$. But with a guide field, this efficient acceleration region is extended along $z$ (as shown in Melzani et al. 2014b), because accelerated particles are guided by the guide field and prevented from being deviated by $B_{x}$. Background particles are more accelerated, and a flatter spectrum is indeed expected.

Because of their larger Larmor radii, background accelerated ions are less affected by the guide field. They present a powerlaw distribution with index $p=8$ for both guide field strengths.

Table 4 shows the energy distribution for the guide field simulations. In both cases, the final kinetic energy is $\sim 44 \%$ of the total initial energy. (We do not include the guide field $B_{y}$ in this initial energy because it cannot be transferred to the particles, and indeed varies by less than a few percentage points during the simulation.) This is less than in the $B_{\mathrm{G}}=0$ case, where this percentage is $62 \%$. The kinetic energy repartition between accelerated ions and electrons is $46 \% / 54 \%$ (ions/electrons) for $B_{\mathrm{G}}=0.5 B_{0}$, and $33 \% / 67 \%$ for $B_{\mathrm{G}}=B_{0}$. This contrasts with the $60 \% / 40 \%$ ratio at $B_{\mathrm{G}}=0$, since here electrons get more energy than do ions.

\section{Summary and discussion}

\subsection{Summary}

We studied the production of high-energy particles by magnetic reconnection in relativistic ion-electron plasmas based on the same 2D PIC simulation data presented by Melzani et al. (2014b). The variety of parameters employed (particle density, temperature, or magnetic field in the background plasma, guide field, mass ratio) allows important aspects of this problem to be grasped. In all cases particles could be divided into two populations that only weakly mix. (i) Particles initially inside the current sheet are trapped inside the magnetic islands as soon as they form during the tearing instability, and remain trapped by the strong circling magnetic structure, even after many island merging events. They are heated by the contraction of the islands. (ii) Particles from the background plasma $E \times B$ drift toward the diffusion region, where either $E<B$ in the no-guide field case or $\boldsymbol{E} \cdot \boldsymbol{B} \neq 0$ in the guide field case allows them to be accelerated directly. As they escape along the reconnection exhaust, they cannot penetrate inside the island because of the strong magnetic structure surrounding them, so they circle at the periphery, where they can gain more energy.

Particles of population (ii) tend to form a power law whenever their magnetization is greater than unity and the inflow Alfvén speed is not too small (Figs. 1 and 3), though sometimes not with a clear and unique slope. The indexes depend on the temperature, particle density, and magnetic field in the background plasma, as well as on the guide field strength. With no guide field, results suggest that the power-law index for species $s$ depends mainly on the background plasma Alfvén speed $V_{\mathrm{A}, \text { in }}^{\mathrm{R}}$, and on the background plasma magnetization for species $s$, independent of whether it is due to the magnetic field strength, a lower particle density, or a relativistic temperature. A higher magnetization leads to a harder power law: for the electrons, $p=-\mathrm{d} \log n(\gamma) / \mathrm{d} \log \gamma=4.5,2.4,2.8,1.7,1.2$ respectively, for magnetizations $\sigma_{\mathrm{e}}^{\text {hot }}=10,27,35,89,260$ (see Table 1). This is expected for reasons exposed in Sect. 4.2.2. These indexes are harder than for collisionless shock acceleration, where $p>2$ (Bell 1978; Sironi \& Spitkovsky 2011b). Ions have a magnetization that is $m_{\mathrm{i}} / m_{\mathrm{e}}$ times less than electrons (for identical or non-relativistic temperatures). As expected, they behave non-relativistically for low magnetizations $\sigma_{i}^{\text {hot }}$ : no power law at $\sigma_{\mathrm{i}}^{\text {hot }}=0.4$; steep ones for $\sigma_{\mathrm{i}}^{\text {hot }}=1.2,3.6(p=8,5.5)$; and beginning of formation of significant power laws at higher magnetization, $p=3.6$ for $\sigma_{\mathrm{i}}^{\text {hot }}=14$, mimicking the values reached for electrons at the same $\sigma_{\mathrm{e}}$. The presence of a weak guide field deforms the power law, and the presence of a strong guide field allows for a slightly harder spectrum (Fig. 3, right) because it allows particles to stay longer in the acceleration region by guiding them in the direction of the reconnection electric field.

The degree of mixing between the two populations (i) and (ii) essentially depends on the ratio of the Larmor radii of the particles in the magnetic field surrounding the islands to the island radius. A hotter background temperature implies more mixing, and so does a weaker guide field. Also, ions have larger Larmor radii and penetrate the islands more easily. This is illustrated in Fig. 5. We stress that even for high background electron temperatures (bottom right panel), the two electron populations remain clearly separated.

The total particle distribution is the sum of populations (i) and (ii), and it depends on their relative importance. We underlined that our simulations are limited in time by the box size. In reality, longer times can be reached and more background particles can be accelerated, so that the background-accelerated population and its power-law nature will dominate in the end. It calls for care when interpreting PIC particle distributions: either very long simulations (also demanding large domains) or the proposed decomposition should be used.

The percentage of magnetic energy converted into kinetic energy is greater at larger inflow magnetization: the final kinetic energy rises to $48 \%, 62 \%, 73 \%$ of the total initial energy, for respective inflow magnetizations $\sigma_{\mathrm{e}}^{\text {hot }}=9.9,83,260$ (see Table 3) at $m_{\mathrm{i}} / m_{\mathrm{e}}=25$. This fraction is lower with a guide magnetic field ( 44\%, Table 4).

The energy distribution between accelerated ions and electrons from the background plasma depends mainly on the strength of the guide magnetic field. With no guide field, it is 


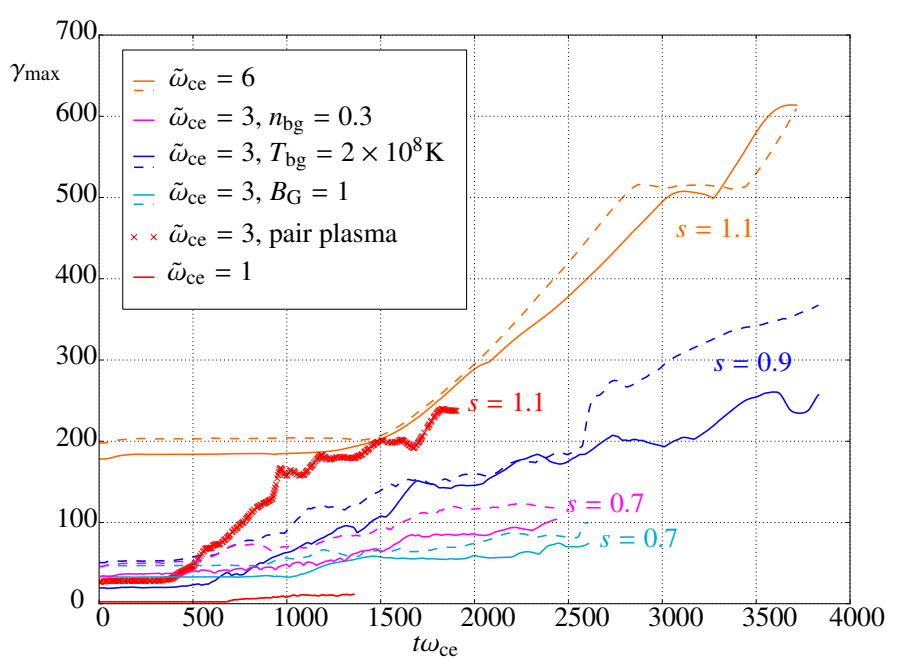

Fig. 4. Maximum Lorentz factor of the background particles for various simulations with $m_{\mathrm{i}} / m_{\mathrm{e}}=25$ or 1 . Solid lines are for electrons, dashed lines for ions and represent $m_{\mathrm{i}} / m_{\mathrm{e}} \times \gamma_{\mathrm{i}, \max }$. Also, $\tilde{\omega}_{\mathrm{ce}}=\omega_{\mathrm{ce}} / \omega_{\mathrm{pe}}$. A $\log -\log$ plot shows that the Lorentz factors grow as $t^{s}$, with the index $s$ shown in the figure.

roughly $60 \%$ for ions and $40 \%$ for electrons, with variations within $3 \%$ when varying the background temperature, magnetization, and Alfvén speed. With a guide field of $0.5 B_{0}$ and $B_{0}$, the ion/electron distribution becomes $46 \% / 54 \%$ and $33 \% / 67 \%$, respectively, with electrons getting more energy than ions. Our conclusion is thus that, overall, ions and electrons are almost equally energized. It is, however, essential to know whether this remains true at realistic mass ratios. With no guide field, our simulations with $m_{\mathrm{i}} / m_{\mathrm{e}}=12$ and $m_{\mathrm{i}} / m_{\mathrm{e}}=50$ showed a variation of $\sim 3 \%$, which cannot be distinguished from the variation due to the different background magnetizations of these simulations. Consequently, and even if higher mass ratios should be tested, it seems that the $\sim 60 \% / 40 \%$ distribution will hold at higher $m_{\mathrm{i}} / m_{\mathrm{e}}$. The case with a guide magnetic field will be explored in more depth in a future work.

The maximum Lorentz factor of the background particles (ii) is shown in Fig. 4. A larger guide field leads to lower the highest Lorentz factors, and this is expected because the reconnection electric field becomes weaker with increasing guide field (Melzani et al. 2014b). A log-log plot shows that the rate of increase is roughly $\gamma_{\max } \propto t^{s}$, with $s$ ranging from 0.7 to 1.1 as the magnetization rises, with identical values for ions and electrons. Particles inside the islands follow the same trend. We stress that this is faster than in collisionless shock acceleration where $\gamma_{\max } \propto t^{0.5}$ (Bell 1978). We also note that the maximal Lorentz factor does not saturate. It should saturate in very large systems when the inter-island distance becomes greater than the particle Larmor radii in the reconnected field $B_{x}$, and when island merging ceases.

\subsection{Discussion}

\subsubsection{Acceleration mechanisms}

The main acceleration mechanism for the background population (ii) is direct acceleration by the reconnection electric field. However, other acceleration mechanisms are present. It can be seen by dividing the background population into several subgroups, each comprising particles in a slab $x_{\min }<x<x_{\max }$ at $t=0$. We can then follow the spatial evolution of these slabs,

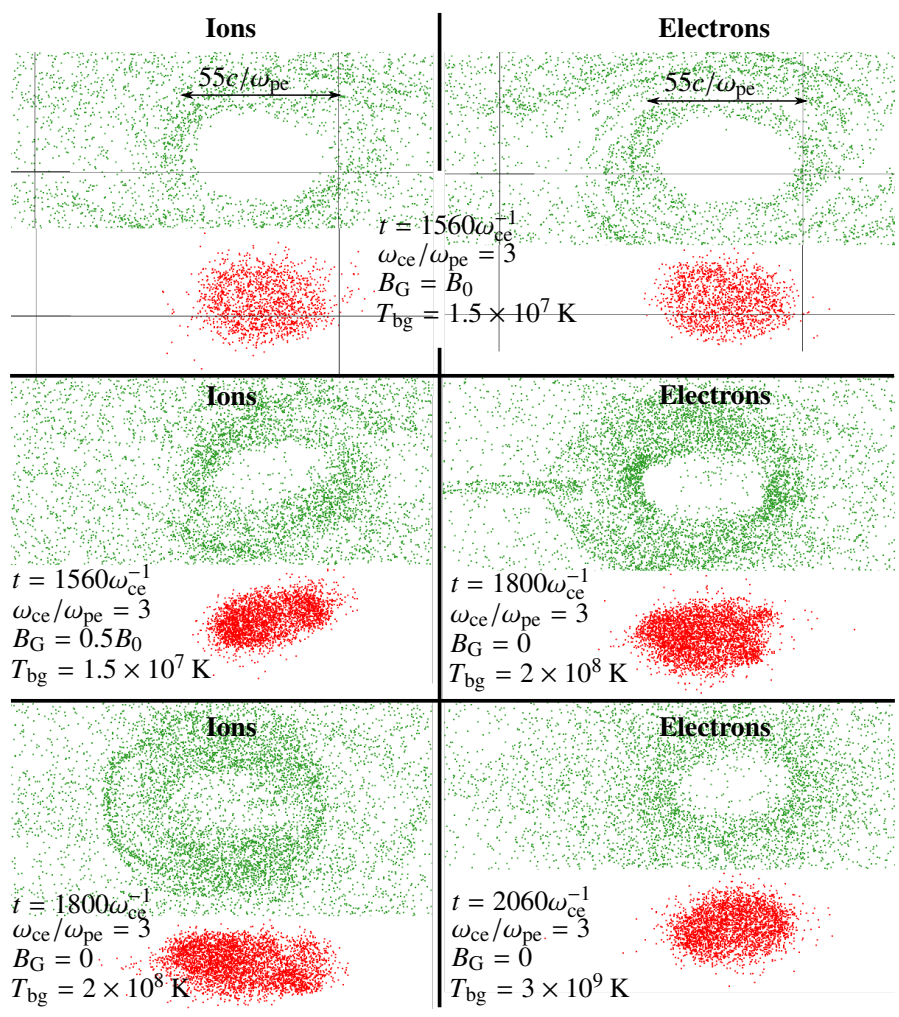

Fig. 5. Particle mixing in the islands. Each panel is a zoom around a magnetic island and shows particles initially outside of the current sheet in green and particles initially located inside the current sheet in red and shifted below for clarity. Each snapshot is taken at the end of the simulations, and these islands result from the merging of many small and then larger islands (from around 20 islands at the end of the linear phase of the tearing instability, to 3 big islands at the end), and yet the two particle populations remain separated. The mass ratio is 25 in all cases.

along with the evolution of their momentum distribution function. After gaining energy in the acceleration region by $E_{\mathrm{rec}}$, background particles escape along the reconnection exhausts and circle at the island's periphery following the strong magnetic field structure. Contraction of the islands (when they merge) creates strong motional electric fields $\boldsymbol{E}=-\boldsymbol{v} \wedge \boldsymbol{B}$ that accelerate these particles. Also, two merging islands create a reconnection event with a reconnection electric field along $+\hat{\boldsymbol{y}}$ that can transfer energy to particles. The combination of these three mechanisms is also reported for pairs by Bessho \& Bhattacharjee (2012) and Sironi \& Spitkovsky (2014).

We find no trace of Fermi acceleration between the converging inflows, as studied analytically or with test particles by Giannios (2010), Kowal et al. (2011), or Drury (2012), because particles cannot cross the current sheet (see Melzani et al. 2014b), but bounce back and forth only inside the diffusion region where they are constantly accelerated by the reconnection electric field. Fermi acceleration is possible only if inflowing particles of species $s$ are energetic enough to cross the diffusion region, i.e., have a Larmor radius $\gamma m_{s} v / e B_{0}$ that is larger than the diffusion zone width $\delta_{s}$. The latter is roughly one inertial length measured at its center (Melzani et al. 2014b), so that the crossing condition can be written $\gamma v / c>\left(\sigma_{s}^{\text {cold }} n_{\mathrm{bg}} / n_{\mathrm{cs}}\right)^{1 / 2}$ (with $\sigma_{s}^{\text {cold }}$ the background magnetization defined by Eq. (3), and $n_{\mathrm{bg}}$ and $n_{\mathrm{cs}}$ the background and current sheet density). The righthand side is greater than unity for a relativistic inflow magnetization. This mechanism thus requires already accelerated particles in the 
Table 5. Order of magnitude for physical parameters in astrophysical environments.

\begin{tabular}{|c|c|c|c|c|c|}
\hline Objects with ion-electron plasmas (with also pairs) & $B(\mathrm{G})$ & $n_{\mathrm{e}}\left(\mathrm{cm}^{-3}\right)$ & $T_{\mathrm{e}}(\mathrm{K})$ & $\sigma_{\mathrm{e}}^{\text {cold }}$ & $V_{\mathrm{A}, \mathrm{in}}^{\mathrm{R}} / c$ \\
\hline Microquasar coronae, X-ray emitting region ${ }^{a}$ & $10^{5}-10^{7}$ & $10^{13}-10^{16}$ & $10^{9}$ & $10^{-1}-10^{5}$ & $0.003-1$ \\
\hline AGN coronae, $X$-ray emitting region ${ }^{b}$ & & & $10^{9}$ & $1.7-180$ & $0.03-0.3$ \\
\hline Giant radio galaxy lobes ${ }^{c}$ & $10^{-6}-10^{-5}$ & $3 \times 10^{-6}$ & $10^{6}$ & $0.8-80$ & $0.02-0.2$ \\
\hline Extragalactic jet, $\gamma$-ray emitting region $(<0.05 \mathrm{pc})^{d}$ & 12 & 80 & & $2 \times 10^{5}$ & $\sim 1$ \\
\hline Extragalactic jet, radio emitting region (kpc scales) ${ }^{e}$ & $1-3 \times 10^{-5}$ & $0.8-5 \times 10^{-8}$ & & $500-2500$ & $\sim 1$ \\
\hline GRB jet, at radius of fast reconnection ${ }^{f}$ & $7 \times 10^{8}$ & $10^{10}$ & $10^{8}$ & $5 \times 10^{12}$ & 0.9 \\
\hline Objects with pair plasmas & $B(\mathrm{G})$ & $n_{\mathrm{e}}\left(\mathrm{cm}^{-3}\right)$ & $T_{\mathrm{e}}(\mathrm{K})$ & $\sigma_{\mathrm{e}}^{\text {cold }}$ & $V_{\mathrm{A}, \mathrm{in}}^{\mathrm{R}} / c$ \\
\hline At the termination shock of pulsa & $10^{4}$ & $0.1-10$ & & $10^{13}$ & $\sim 1$ \\
\hline In a pulsar wind nebulae ${ }^{h}$ & $5 \times 10^{-3}$ & 5 to $10^{3}$ & $\gamma \sim 10-10^{9}$ & $<0.5$ & 0.6 \\
\hline
\end{tabular}

Notes. ${ }^{(a)}$ Analytical disk and corona models, de Gouveia dal Pino \& Lazarian (2005); Di Matteo (1998); Merloni \& Fabian (2001); Reis \& Miller (2013); matching observed spectra with radiation models, Del Santo et al. (2013); Romero et al. (2014). ${ }^{(b)}$ Analytical disk and corona models, Di Matteo (1998); Merloni \& Fabian (2001); Reis \& Miller (2013). ${ }^{(c)}$ Observations, Kronberg et al. (2004). ${ }^{(d)}$ Analytical model assuming $\sigma_{\mathrm{i}}^{\text {cold }}=100$, Giannios et al. (2009). See also Giroletti et al. (2004) for magnetic field measurements (0.2 G, but on larger scales). ${ }^{(e)}$ Observations, Schwartz et al. (2006). See also Romanova \& Lovelace (1992). ${ }^{(f)}$ Analytical model, McKinney \& Uzdensky (2012). Pairs are also present, with $n_{\text {pair }} \sim 10 n_{\mathrm{e}}{ }^{\left({ }^{g}\right)}$ Analytical model and observations, Bucciantini et al. (2011); Sironi \& Spitkovsky (2011a). ${ }^{(h)}$ Analytical model and observations, Atoyan \& Aharonian (1996); Meyer et al. (2010); Uzdensky et al. (2011); Cerutti et al. (2013). The plasma distribution function is a broken power law with Lorentz factors $\gamma$ in the indicated range. We note that Cerutti et al. (2013) considers only the high-energy electron population, so have higher magnetizations.

inflow, which is possible for very high temperatures, or for an out-of-equilibrium component pre-accelerated by other mechanisms outside of the diffusion region such as neighboring reconnection sites or large scale flow turbulence (see Sect. 4.3.3). We have neither of these in our simulations, and their presence in real situations should be addressed.

We now turn to the building of the power-law spectrum in our simulations. The basic idea is that particles enter the acceleration region at all distances from the central X-point, with those entering near the center being deviated toward the exhausts by $B_{x}$ more slowly than those entering at the edges. They thus gain more energy, and a monoenergetic inflow is transformed into a broader distribution. The ingredient to build a power law, underlined by Zenitani \& Hoshino (2001), is that relativistic particles have a Larmor radius that increases with their Lorentz factor: high-energy particles rotate slowly in $B_{x}$ and are held in the acceleration region even longer as they are accelerated, thus facilitating the creation of hard tails. With this argument alone, Zenitani \& Hoshino (2001) predict a power law with in$\operatorname{dex} p \propto c B_{x} / E_{\mathrm{rec}}$, but their model is very simple. Bessho \& Bhattacharjee (2012) analytically derive the spectra of particles accelerated by $E_{\text {rec }}$ and escaping from the X-point to find $\mathrm{d} n / \mathrm{d} \gamma \propto \gamma^{-1 / 4} \exp \left\{-a \gamma^{1 / 2}\right\}$. It is a power law with an exponential cutoff, occurring at higher energies for relativistic X-points because $a \propto c B_{x} / E_{\text {rec }}$. In the end, the X-point accelerated particles gain more energy around the contracting islands and during reconnection events between merging islands, to result in the distribution that we show in this paper.

\subsubsection{Condition for hard high-energy tails}

It appears from our data that the hardness of the energy distribution for species $s$ is controlled by its background magnetization $\sigma_{s}^{\text {hot }}$ and by the Alfvén speed $V_{\mathrm{A} \text {,in }}^{\mathrm{R}}$. The power law is harder for higher magnetizations (Table 2). For a fixed magnetization $\sigma_{\mathrm{e}}^{\text {hot }}=83$, the simulations with $m_{\mathrm{i}} / m_{\mathrm{e}}=1,12$, and 25 feature different inflow Alfvén speeds $V_{\mathrm{A}, \text { in }}^{\mathrm{R}}=0.988,0.93$, and 0.88 , and also different power-law indexes for electrons: $p=1.5$,
1.6, 1.8, respectively. Similarly, when compared to our work at a given electron magnetization, the PIC simulations of Sironi \& Spitkovsky (2014) for pair plasmas present harder distributions, simply because with pairs and a given $\sigma_{\mathrm{e}}$, the Alfvén speed is higher. A higher inflow Alfvén speed thus leads to harder distributions.

This can be interpreted as follows. The building of a highenergy tail for species $s$ requires two ingredients: an inflow magnetization $\sigma_{s}>1$ so that the transfer of magnetic energy can exceed the particles' rest mass, and thus accelerate them to relativistic energies; and a large enough ratio $E_{\text {rec }} /\left(c B_{0}\right)$ to have a hard distribution. The latter condition roughly states that the residence time $\gamma m_{s} /\left(q_{s} B_{x}\right)$ of a particle in the acceleration region must be longer than its acceleration time $\gamma m_{s} c /\left(q_{s} E_{\mathrm{rec}}\right)$ by $E_{\mathrm{rec}}$. This is supported by the analytical models of Zenitani \& Hoshino (2001) and Bessho \& Bhattacharjee (2012) cited above. Given that the reconnection rate $^{1} E^{*}=E_{\mathrm{rec}} /\left(B_{0} V_{\mathrm{A}, \text { in }}^{\mathrm{R}} \cos \theta\right)$ lies in a narrow range for various simulations $\left(E^{*} \sim 0.14-0.30\right.$, Melzani et al. 2014b), the condition of a large ratio $E_{\text {rec }} /\left(c B_{0}\right)=$ $E^{*} V_{\mathrm{A} \text {,in }}^{\mathrm{R}} \cos \theta / c$ consequently translates into a relativistic inflow Alfvén speed $V_{\mathrm{A} \text {,in }}^{\mathrm{R}}$ and into not too strong a guide field $(\cos \theta=$ $\left.\left(1+B_{\mathrm{G}}^{2} / B_{0}^{2}\right)^{-1 / 2}\right)$.

For the ions, a hard distribution requires $\sigma_{\mathrm{i}}^{\text {hot }} \gg 1$ and $V_{\mathrm{A} \text {,in }}^{\mathrm{R}} \sim c$. But the condition $V_{\mathrm{A}, \text { in }}^{\mathrm{R}} \sim c$ is equivalent to a total magnetization $\sigma_{\mathrm{i}+\mathrm{e}}\left(B_{\mathrm{tot}}\right)>1$ (Eq. (5)), which is already fulfilled if $\sigma_{\mathrm{i}}^{\text {hot }} \gg 1$. For the electrons, a hard distribution requires $\sigma_{\mathrm{e}}^{\text {hot }} \gg 1$ and $V_{\mathrm{A}, \text { in }}^{\mathrm{R}} \sim c$. The latter translates to $\sigma_{\mathrm{i}+\mathrm{e}}\left(B_{\mathrm{tot}}\right)>1$, so that we have (if we neglect temperature effects) $\sigma_{\mathrm{e}}^{\text {cold }} \sim$ $\left(1+m_{\mathrm{i}} / m_{\mathrm{e}}\right) \sigma_{\mathrm{i}+\mathrm{e}}>1+m_{\mathrm{i}} / m_{\mathrm{e}} \sim 2000$. Here the condition on the Alfvén speed is consequently more stringent than the condition on the magnetization. We conclude that hard ion distributions are obtained when $\sigma_{i}^{\text {hot }} \gg 1$ and hard electron distributions when $V_{\mathrm{A} \text {,in }}^{\mathrm{R}} \sim c$.

1 The residence time $\gamma m_{s} /\left(q_{s} B_{x}\right)$ implies the reconnected field $B_{x}$, and not the inflowing component $B_{0}$ as in the reconnection rate. However, the ratio $B_{0} / B_{x}$ is roughly the same for all magnetizations and all simulations. 
We finally point out that the Alfvén speed, and thus also the ratio $E_{\text {rec }} / B_{0}$, saturates at $c$ for strong magnetizations. We consequently expect the particle distribution hardness to also saturate to some value with, as indicated by our simulations, a power-law index $p$ slightly below 1.2. Reconnection in environments with $\sigma_{i}^{\text {hot }} \gg 1$ should thus produce ion and electron distributions with power-law index $p \lesssim 1.2$.

\subsection{Astrophysical outlook}

\subsubsection{Objects and orders of magnitude}

Magnetic reconnection can play a major role for four particular purposes: high-energy flare production, steady emission of radiation, large scale outflow launching, and plasma heating or non-thermal particle production. We discuss which objects these phenomena are observed in, give order of magnitudes for the main parameters, and point out where our work is applicable. Since our study concerns relativistic ion-electron reconnection, we restrict the discussion to environments where relativistic ionelectron plasmas are expected. Table 5 summarizes the physical conditions encountered in the objects mentioned here.

Flare-like emission of high-energy photons is observed, for example, in the $\gamma$-ray region of AGN jets (Giannios 2010) or in microquasar and AGN coronae (Di Matteo 1998; Merloni \& Fabian 2001; Reis \& Miller 2013). Concerning AGN jets, flares in the $\mathrm{GeV}-\mathrm{TeV}$ range are observed and may come from close to the AGN ( $<0.05$ pc, Giroletti et al. 2004). There, Giannios et al. (2009) assume an ion magnetization $\sigma_{i}^{\text {cold }} \sim 100$, which gives an Alfvén speed $\sim c$ (Table 5, line d). Concerning the coronae of AGNs and microquasars, various models constrained by observations predict ion magnetizations in the range $\sigma_{\mathrm{i}}^{\text {cold }} \sim 10^{-5}-10^{2}$ (Table 5, lines a and b). In the most magnetized case, the Alfvén speed is $\sim c$. According to our results, reconnection in such environments should produce electron distributions with hard tails $(p \sim 1)$, and if we apply our results for electrons to the ions, the latter should also form hard tails $(p \sim 2)$. Quite generally, high-energy flares can be explained by reconnecting events under three conditions: the large scale magnetic field must possess enough energy and the large scale flow or the flow turbulence must lead to enough reconnection events; the reconnection process must produce high-energy particles with hard distributions; these high-energy particles must be able to radiate. The first point is linked to the large scale properties of the object and can be investigated with simulations on large scales, while the last two points concern microphysics and must be addressed with first principle simulations. We believe we have answered the second point: magnetic reconnection does produce hard highenergy distributions whenever the ion magnetization is above unity, which can indeed be the case in the environments mentioned above. Concerning photon emission, we expect particles trapped inside the islands (population i) to produce mostly thermal synchrotron-Bremsstrahlung. On the other hand, particles accelerated at the X-point (population ii) are likely to radiate collimated beams when suddenly encountering the strong magnetic field structure at the end of the exhausts at the island entrance. This was demonstrated in the no-guide field case by Cerutti et al. (2012b) in pair plasmas, and should also hold for ion-electron plasmas because the overall magnetic structure is not too different. The radiation spectrum is then anisotropic and reaches energies on the order of $\gamma^{2} \omega_{\text {ce }}$ with $\gamma$ the particles Lorentz factor and $\omega_{\mathrm{ce}}=e B / m_{\mathrm{e}}$. With a guide field, radiation should occur together with particle acceleration in the $\boldsymbol{E} \cdot \boldsymbol{B} \neq 0$ area, because particles then swirl around the guide field. It should consequently be more regular and less flare-like (Cerutti et al. 2013). Since reconnection with a guide field is more generic in the complex magnetic field structures of coronae or jets than antiparallel reconnection, the question of photon emission in such a case is very relevant.

Magnetic reconnection can produce radiation in a wide range of frequencies, which for the synchrotron component depends on the strength of the reconnecting magnetic field. An example is the radio emission, on kilo-parsec scales, of extragalactic jets. Explanation of these spectra can invoke magnetic reconnection events, in particular, to explain the hard photon indexes (Romanova \& Lovelace 1992). Observations indicate electron magnetizations in the range $\sigma_{\mathrm{e}}^{\text {cold }} \sim 500-2500$ (Table 5, line e), which corresponds to ion magnetizations $\sigma_{\mathrm{i}}^{\text {cold }} \sim 0.3-1.3$ and to Alfvén speeds $\sim 0.5-0.8 c$. This is between the two cases $\omega_{\text {ce }} / \omega_{\text {pe }}=1$ and $\omega_{\text {ce }} / \omega_{\text {pe }}=3$ of our study, for which the kinetic energy takes a large amount of the magnetic energy (between $45 \%$ and $60 \%$, Tables 3 and 4), and is distributed as $60 / 40 \%$ to $30 / 70 \%$ between ions and electrons, respectively, depending on the guide field strength. Most importantly, for these parameters the background accelerated electrons form power laws with indexes between 4.5 and 1.5 (Table 2 and Fig. 3), which can then indeed emit hard spectra.

Large scale magnetic reconnection events may be at the origin of large scale transient jets in microquasar systems (de Gouveia dal Pino \& Lazarian 2005; de Gouveia Dal Pino et al. 2010; Kowal et al. 2011; McKinney et al. 2012; Dexter et al. 2014), for example via a magnetic field reversal accreted onto a magnetically arrested disk. Reconnection then occurs in the accretion disk coronae, near the black hole, where particle densities and magnetic fields are high. de Gouveia dal Pino \& Lazarian (2005) estimate that the ion magnetization close to the black hole is $\sigma_{\mathrm{i}}^{\text {cold }} \sim 60$, i.e., $\sigma_{\mathrm{e}}^{\text {cold }} \sim 10^{5}$ (Table 5, line a).

Magnetic reconnection can also efficiently heat the plasma by dissipating the magnetic field energy. This is invoked for the heating of AGNs and microquasar coronae, which must reach electron temperatures on the order of $10^{9} \mathrm{~K}$ to be able to inverseComptonize seed photons to X-ray energies. In microquasars, a coronal population of non-thermal high-energy electrons is also required by the observation of $\mathrm{MeV}$ photons (Poutanen \& Veledina 2014). Alfvén speeds in these coronae are estimated to be on the order of $0.003 c-c$ (Table 5 , line a). In our corresponding simulations, the final kinetic energy is a large fraction of the initial magnetic energy $\left(48 \%\right.$ at $V_{\mathrm{A}}=0.5 c$, up to $75 \%$ at $V_{\mathrm{A}}=0.88 c$, Table 3 ), thus allowing an efficient energy transfer to the plasma. For this range of Alfvén speeds, accelerated electron distributions can be steep $\left(p>4\right.$ for $\left.V_{\mathrm{A}}=0.5 c\right)$ or hard $\left(p \sim 1.2\right.$ for $\left.V_{\mathrm{A}}=0.97 c\right)$, and in the latter case reconnection can indeed produce a non-thermal population. An important point is the question of the energy distribution between ions and electrons. Our results show that energy is almost equally distributed between ions (60\% to $30 \%$ ) and electrons (40\% to $70 \%)$, so that we do not expect large temperature differences (as studied in some models, Di Matteo et al. 1997) from this heating mechanism. Other models of the $\mathrm{MeV}$ component from microquasars invoke the emission of electrons in the jet and require hard electron distributions with indexes $p \sim 1.5$ (Zdziarski et al. 2014). The ion magnetization in these models needs to be greater than unity. Our work demonstrates that magnetic reconnection in these conditions can provide these hard electron spectra. Also, very similar conditions are expected in the lobes of radio galaxies $\left(V_{\mathrm{A}} \sim 0.02-0.2 c\right.$, Kronberg et al. 2004), and our conclusions also apply there, especially for the ion/electron energy distribution. 
A final application concerns the extraterrestrial $\mathrm{PeV}$ neutrinos detected by IceCube (IceCube Collaboration 2013). They can come from the photopion $(p \gamma)$ interaction of high-energy protons or ions produced by high-energy machines (such as GRBs, Petropoulou et al. 2014). The ability of magnetic reconnection to accelerate ions in highly magnetized environments is then very relevant. We find that the highest Lorentz factor for ions follows the same trend as for electrons $\left(\gamma \propto t^{s}\right.$, $s \sim$ 0.7-1.1), and for ion magnetization $\sigma_{\mathrm{i}} \gg 1$, we expect ions to feature the same power-law spectra as electrons, with a slope $p \lesssim 1.2$.

\subsubsection{Scaling of the results, importance of radiative braking, Compton drag and pairs}

The reconnection physics with the Harris geometry described in this paper only depends on the inflow plasma magnetization and temperature and not on the absolute values of magnetic field and particle number density. For example, reconnection in a microquasar corona close to the hole and in the $\gamma$-ray emitting region of an extragalactic jet takes place with the same magnetizations (Table 5) and thus features the same reconnection rate, particle spectra, or energy distribution, even if magnetic field strengths differ by six orders of magnitude (provided, however, that the geometry is the same). This is true as long as reconnection occurs in the collisionless regime, an issue discussed, for example, by Goodman \& Uzdensky (2008) and Ji \& Daughton (2011). Also, it requires that effects such as radiative braking by emission or Compton drag, or pair annihilations, do not perturb the reconnection physics. Such effects imply the actual values of magnetic field and particle number densities and can lead to very different physics for the same magnetization. To evaluate this, we estimate in Appendix A when an electron loses a significant fraction of its energy during any given time or over a length scale that is dynamically important for the magnetic reconnection physics. These time and length scales are taken as a cyclotron or plasma period or as an inertial scale. Particles will eventually radiate and cool farther away, but with no influence on the reconnection physics. We summarize the main points of this appendix here.

Radiative braking due to synchrotron radiation remains negligible on cyclotron scales as long as $(\gamma / 100)^{2} B<10^{11} \mathrm{G}$ (Eq. (A.1)), where $\gamma$ is the Lorentz factor of an electron. This is negligible for all objects of Table 5, except in the pulsar wind nebulae where $\gamma$ can reach $10^{9}$ (Meyer et al. 2010). Radiative braking due to Coulomb collisions can be estimated by assuming a thermal Bremsstrahlung and remains negligible on inertial length scales as long as $\left(T_{\mathrm{e}} / m_{\mathrm{e}} c^{2}\right)^{1 / 2}\left(n_{\mathrm{e}} / 5 \times 10^{12} \mathrm{~cm}^{-3}\right)^{3 / 2}<1$ (Eq. (A.2)). This is the case for objects of Table 5, except in microquasar coronae close to the black hole where $n_{\mathrm{e}}$ is high.

Compton drag does not affect the electron dynamics on inertial length scales as long as $(\gamma / 100)\left(1 \mathrm{~cm}^{-3} / n_{\mathrm{e}}\right)^{1 / 2} U_{\mathrm{ph}}<$ $10^{10} \mathrm{erg} / \mathrm{cm}^{3}$, with $U_{\mathrm{ph}}$ the radiation field energy density (Eq. (A.3)). For a blackbody spectrum, Compton drag is insignificant for photon temperatures $T_{\mathrm{ph}}<10^{6} \mathrm{~K}$. Consequently, objects with electron temperatures $T_{\mathrm{e}}>10^{6} \mathrm{~K}$ on scales large enough that the optical depth is important and photons are thermalized are in the range where Compton drag is efficient. It should be noted that the electrons locally heated by the magnetic reconnection cannot thermalize the radiation, because the reconnection region is optically thin (Eq. (A.4)). Alternatively, the photon energy density $U_{\text {ph }}$ produced by the magnetic reconnection can be computed from the synchrotron or Bremsstrahlung emissivity, assuming that the emission takes place over a volume $\left(a d_{\mathrm{e}}\right)^{3}$ with $d_{\mathrm{e}}$ the electron inertial length and $a$ a geometrical factor. Compton drag against the synchrotron photons is negligible as long as $a(B / 1 G)^{2}(\gamma / 100)^{3}<$ $10^{36}$ (Eq. (A.6)), and that due to Bremsstrahlung emission as long as $a\left(T_{\mathrm{e}} / 10^{8} \mathrm{~K}\right)^{1 / 2}\left(n_{\mathrm{e}} / 1 \mathrm{~cm}^{-3}\right)(\gamma / 100)<10^{38}$ (Eq. (A.8)). Compton drag by these photon fields is thus negligible for all objects of Table 5. The radiation field can also originate outside of the reconnection region. For example, at a distance $R$ from an object emitting at a fraction $\alpha$ of the Eddington luminosity, the photon energy density can be estimated as $U_{\mathrm{ph}}=$ $\alpha\left(R_{\mathrm{S}} / R\right)^{2}\left(10 \mathrm{~km} / R_{\mathrm{S}}\right) \times 10^{15} \mathrm{erg} / \mathrm{cm}^{3}$, with $R_{\mathrm{S}}$ the Schwarzschild radius of the object. The threshold where Compton drag against these photons is significant can be reached in the inner parts of microquasar coronae if $n_{\mathrm{e}}$ is not too high (as given by Eq. (A.10)) (see also Goodman \& Uzdensky 2008).

Finally, photons of energy above $m_{\mathrm{e}}^{2} c^{4} / \epsilon_{0}$ can annihilate with ambient photons (of typical energy $\epsilon_{0}$ ) to produce pairs. This can be the case if $B(\gamma / 100)^{2}>2 \times 10^{9} \mathrm{G}$ for high-energy synchrotron photons or if $T_{\mathrm{e}}>10^{9} \mathrm{~K}$ for Bremsstrahlung (Eqs. (A.11) and (A.12)). Inverse Compton events can also produce such photons if the electron Lorentz factors are $\gamma>m_{\mathrm{e}} c^{2} / \epsilon_{0}$ (see Appendix A).

In any case, pair creation will disturb the reconnection dynamic only if the creation occurs inside or close to the reconnection region. The mean-free-path $l_{\gamma \gamma}$ of high-energy photons should thus be compared to an inertial length $d_{\mathrm{e}}$. For a blackbody gas of photons at temperature $T_{\mathrm{ph}}$, we have $l_{\gamma \gamma, \mathrm{BB}} / d_{\mathrm{e}}=$ $\left(n_{\mathrm{e}} / 1 \mathrm{~cm}^{-3}\right)^{1 / 2}\left(10^{6} \mathrm{~K} / T_{\mathrm{ph}}\right)^{3}$ (Eq. (A.14)). However, as underlined previously, a blackbody spectrum of photons is not easy to achieve. If photons cannot be thermalized, then the $\gamma \gamma$ opacity must be computed from the rate of production of photons by synchrotron and Bremsstrahlung radiation in the reconnection region. Concerning Bremsstrahlung emission, we find with Eq. (A.19) that pairs form far away from the reconnection region for all objects of Table 5. Concerning synchrotron emission, we find with Eq. (A.17) that for radio lobes, radio emitting regions of extragalactic jets or pulsar wind nebulae, $l_{\gamma \gamma \text {,sync }} \gg d_{\mathrm{e}}$ holds, so that pairs form far away from the reconnection site. For microquasar coronae close to the hole, for extragalactic jet $\gamma$-ray regions, for GRB jets, or for pulsar wind termination shocks, however, we have $l_{\gamma \gamma \text {,sync }} \ll d_{\mathrm{e}}$, and pairs form inside the reconnection region. For microquasars, the photon field can also be deduced from the energy density at a distance $R$ from an object emitting at a fraction of the Eddington luminosity. We find with Eq. (A.21) that pair production can be significant for high luminosities, small radii, and not too high particle densities. Such a regime is studied by Uzdensky (2011).

\subsubsection{Other acceleration sites during reconnection}

In this paper, we have investigated particle acceleration in or close to the diffusion region. Other energy conversion locations exist around reconnection sites. One is along the magnetic separatrices far downstream of the diffusion region (observed, e.g., at the magnetopause: Khotyaintsev et al. 2006) on length scales of hundreds of ion inertial lengths. There, magnetic energy conversion occurs as the plasma flows through the complex structure of collisionless nonlinear waves (slow shock(s), compound wave, and rotational wave). Instabilities and parallel electric fields in these regions can produce thermal and non-thermal electrons (Drake et al. 2005; Egedal et al. 2009, 2012). This shock structure has been investigated in the non-relativistic case (Liu et al. 2012; Higashimori \& Hoshino 2012). In a relativistic 
situation, the different phase speeds of the waves may lead to different results. Of particular interest is the energy distribution between bulk, thermal, ion, and electron components, and its importance relative to the locations discussed here.

Another site for particle acceleration is at the dipolarization front (Vapirev et al. 2013), where the first reconnected field lines are swept away and drag the ambient plasma. Such a situation is prone to instabilities and particle acceleration. Also, turbulence associated with magnetic reconnection can lead to particle acceleration via a second-order Fermi process.

We emphasize, however, that high-energy particle production in and near the diffusion zone, directly by the reconnection electric field as discussed in the present manuscript, should be very important for relativistic inflow magnetizations because the electric field is very large $\left(E_{\mathrm{rec}} / B_{0} \sim 0.2 V_{\mathrm{A} \text {,in }}^{\mathrm{R}} \sim 0.2 c\right.$, see Melzani et al. 2014b).

\subsubsection{Open questions}

The present study offers useful insights into the properties of magnetic reconnection, but remains simplified in many respects. Reconnection configurations in real environments are likely to often involve guide fields, but also asymmetric plasmas and fields from each side of the current sheet (Aunai et al. 2013; Eastwood et al. 2013), or normal magnetic fields (i.e., along $\hat{\boldsymbol{x}}$ here) owing to the ambient field (e.g., for the magnetotail, Pritchett 2005a). Magnetic reconnection is also likely to be forced by external plasma and field line motions (Lyubarsky $\&$ Liverts 2008). The reconnection electric field is then imposed by the forcing (Pei et al. 2001; Pritchett 2005b; Ohtani \& Horiuchi 2009; Klimas et al. 2010) and can be larger than in the spontaneous case. Particle acceleration can consequently be enhanced. The initial equilibrium can also have an impact on the late evolution and particle distributions. Study of 2D situations, such as X-point collapse or force-free equilibrium (e.g., Graf von der Pahlen \& Tsiklauri 2014; Liu et al. 2014), shows few differences with the Harris case, and we expect them to produce the same kind of distributions. However, full 3D initial configurations can lead to very different outcomes, as suggested by the few existing kinetic studies (Baumann \& Nordlund 2012; Olshevsky et al. 2013). Reconnection and particle acceleration at 3D nulls or at quasi separatrix layers (Pontin 2011) deserves further research.

A crucial question concerns the validity of our results in a real 3D reconnection event. Magnetic islands then become extended filaments, modulated or broken by instabilities in the third dimension or by a lack of coherence of the tearing instability (Jaroschek et al. 2004; Zenitani \& Hoshino 2008; Daughton et al. 2011; Liu et al. 2011; Kagan et al. 2013; Markidis et al. 2013). For this reason we may expect more particle mixing, but current sheet particles may also still be trapped in the strong magnetic structure surrounding the filaments. Particle acceleration at X-points may also be disturbed. However, 3D results in pair plasmas by Sironi \& Spitkovsky (2014) are encouraging since they show that energization is still efficient and leads to power-law tails with similar indexes, essentially because the small scale physics around the X-point and during filament mergings is the same as in $2 \mathrm{D}$.

There is also a strong need to understand the interplay between large and small scales better. Coronal heating by reconnection and large scale outflow launching are cases where the large scale flow sets the conditions for the occurrence of reconnection, which in turn largely modifies the large scale flow conditions. For example, Jiang et al. (2014) show that global simulations of the formation of an accretion disk corona requires understanding the role of reconnection in the MRI turbulence. Shocks, possibly collisionless, are also fundamental microphysical processes that shape the flow on all scales of accreting black holes (Walder et al. 2014). Daldorff et al. (2014) illustrate the power of a coupled MHD/PIC approach with a simulation of the Earth's magnetosphere.

Finally, we emphasize that the ability of magnetic reconnection to accelerate protons or heavier ions is a key question, first because they can produce mesons and then pairs, which can lead to a different photon spectrum, and second because this channel can produce neutrinos, and characterizing the neutrino spectrum from high-energy objects is compulsory for distinguishing it from those predicted by dark matter models. With high-energy extraterrestrial neutrinos now being detected (IceCube Collaboration 2013), this is a very exciting perspective.

Acknowledgements. We thank the referee for comments that helped to improve the present work. This work was performed using HPC ressources from GENCICCRT/IDRIS/CINES/TGCC (grant x2013046960). Tests were conducted at the Pôle scientifique de modélisation numérique, PSMN, at the ENS Lyon. We acknowledge the Programme National Hautes Énergies (PNHE) for financial support.

\section{Appendix A: The importance of radiative braking, Compton drag, and pair creations}

Two relevant issues are the importance of radiative braking and of pair creation. We first investigate radiative braking of electrons and then study the opacity of high energy photons to $\gamma \gamma$ annihilations.

\section{Electron braking by emission of radiation or by Compton drag}

Electrons lose energy by emitting photons when being scattered by magnetic fields (synchrotron-like radiation) or by Coulomb collisions (Bremsstrahlung-like radiation) or when colliding with photons (inverse-Compton events). For the synchrotron component, the energy $\delta E_{\text {sync }}$ lost by an electron of Lorentz factor $\gamma$ and velocity $\beta c$, gyrating in a magnetic field $B$, averaged over pitch angles, and during one cyclotron orbit, is (Rybicki \& Lightman 1979)

$\frac{\delta E_{\mathrm{sync}}}{\gamma m_{\mathrm{e}} c^{2}}=\frac{8 \pi}{9} \beta^{2} \gamma^{2} \frac{r_{0} \omega_{\mathrm{ce}}}{c}=\frac{B}{1.4 \times 10^{11} \mathrm{G}}\left(\frac{\gamma}{100}\right)^{2}$,

with $r_{0}$ the classical electron radius and $\omega_{\mathrm{ce}}=e B / m_{\mathrm{e}}$. On the other hand, electron cooling by Coulomb collisions can be evaluated via the thermal Bremsstrahlung emission formula, giving an energy $\delta E_{\mathrm{Brem}}$ lost during one plasma period $\omega_{\mathrm{pe}}^{-1}$ :

$\frac{\delta E_{\mathrm{Brem}}}{m_{\mathrm{e}} c^{2}}=\left(\frac{T_{\mathrm{e}}}{m_{\mathrm{e}} c^{2}}\right)^{1 / 2}\left(\frac{n_{\mathrm{e}}}{5 \times 10^{12} \mathrm{~cm}^{-3}}\right)^{3 / 2}$.

It shows that synchrotron braking is not relevant for the objects of Table 5, except for pulsar wind nebulae and very high Lorentz factor electrons, while braking by Bremsstrahlung emission is significant for reconnection in microquasar magnetospheres close to the black hole. 
The last braking mechanism is inverse-Compton scattering of ambient photons by electrons. The energy $\delta E$ lost by an electron during one plasma period $\omega_{\mathrm{pe}}^{-1}$ is at most

$$
\begin{aligned}
\frac{\delta E_{\mathrm{IC}}}{\gamma m_{\mathrm{e}} c^{2}} & =\frac{4}{3} \frac{\sigma_{\mathrm{T}} c \beta^{2} \gamma}{\omega_{\mathrm{pe}}} \frac{U_{\mathrm{ph}}}{m_{\mathrm{e}} c^{2}} \\
& =\frac{\gamma \beta^{2}}{100}\left(\frac{1 \mathrm{~cm}^{-3}}{n_{\mathrm{e}}}\right)^{1 / 2} \frac{U_{\mathrm{ph}}}{1.7 \times 10^{10} \mathrm{erg} / \mathrm{cm}^{3}},
\end{aligned}
$$

where $U_{\mathrm{ph}}$ is the photon energy density and $\sigma_{\mathrm{T}}$ Thomson cross section. For a blackbody radiation, the energy density is given by $U_{\mathrm{ph}}=\left(\pi^{2} / 15\right) T_{\mathrm{ph}}^{4} /(\hbar c)^{3}$. It reaches the density $1.7 \times 10^{10} \mathrm{erg} / \mathrm{cm}^{3}$ for $T_{\mathrm{ph}}=1.2 \times 10^{6} \mathrm{~K}$. Below this temperature, electrons do not significantly lose energy by Compton drag, while above they do.

However, it should be noted that a blackbody radiation at $T_{\mathrm{ph}}$ requires the thermalization of the photons produced by the hot electrons, a fact impossible to achieve on an inertial length scale $d_{\mathrm{e}}$ given that the mean-free-path to Compton scattering is

$\frac{l_{\text {Compt }}}{d_{\mathrm{e}}}=\frac{1}{d_{\mathrm{e}} \sigma_{\mathrm{T}} n_{\mathrm{e}}}=\left(\frac{9 \times 10^{36} \mathrm{~cm}^{-3}}{n_{\mathrm{e}}}\right)^{1 / 2}$,

and would thus imply densities of $n_{\mathrm{e}} \sim 10^{36} \mathrm{~cm}^{-3}$. The radiation produced by the hot electrons in the reconnection region consequently escapes from this region before being thermalized. If we assume that the photons are produced over an optically thin volume $\left(a d_{\mathrm{e}}\right)^{3}$, with $a$ a geometrical factor, and that the emissivity is $\mathrm{d} W_{\mathrm{ph}} / \mathrm{d} t \mathrm{~d} V$, then the energy density of the gas of photons is $U_{\mathrm{ph}} \sim a d_{\mathrm{e}} / c \times \mathrm{d} W_{\mathrm{ph}} / \mathrm{d} t \mathrm{~d} V$. Expressions for $U_{\mathrm{ph}}$ should thus be obtained for synchrotron and Bremsstrahlung radiations.

Concerning synchrotron radiation, the total power emitted by an electron is $P_{\text {emit,sync }}=(4 / 9) r_{0}^{2} c \beta^{2} \gamma^{2} B^{2}$, and the emissivity is $\sim n_{\mathrm{e}} P_{\text {emit,sync, }}$, so that

$$
\begin{aligned}
U_{\mathrm{ph}, \mathrm{sync}}= & 9 \times 10^{-26} \mathrm{erg} / \mathrm{cm}^{3} \\
& \times a\left(\frac{B}{1 \mathrm{G}}\right)^{2}\left(\frac{n_{\mathrm{e}}}{1 \mathrm{~cm}^{-3}}\right)^{1 / 2}\left(\frac{\gamma \beta}{100}\right)^{2} .
\end{aligned}
$$

This expression can be inserted into Eq. (A.3), to yield

$$
\frac{\delta E_{\mathrm{IC}, \mathrm{sync}}}{\gamma m_{\mathrm{e}} c^{2}}=5.3 \times 10^{-36} \times a\left(\frac{B}{1 \mathrm{G}}\right)^{2} \beta\left(\frac{\gamma \beta}{100}\right)^{3},
$$

which is always well below unity for the objects of Table 5 .

As for Bremsstrahlung radiation, we use the emissivity for a thermalized plasma at temperature $T_{\mathrm{e}}$, to find

$$
\begin{aligned}
U_{\mathrm{ph}, \text { Brem }}= & 2.5 \times 10^{-28} \mathrm{erg} / \mathrm{cm}^{3} \\
& \times a\left(\frac{T_{\mathrm{e}}}{10^{8} \mathrm{~K}}\right)^{1 / 2}\left(\frac{n_{\mathrm{e}}}{1 \mathrm{~cm}^{-3}}\right)^{3 / 2} .
\end{aligned}
$$

Once inserted into Eq. (A.3), it yields

$$
\frac{\delta E_{\mathrm{IC}, \text { Brem }}}{\gamma m_{\mathrm{e}} c^{2}}=1.5 \times 10^{-38} \times a\left(\frac{T_{\mathrm{e}}}{10^{8} \mathrm{~K}}\right)^{1 / 2}\left(\frac{n_{\mathrm{e}}}{1 \mathrm{~cm}^{-3}}\right) \frac{\gamma \beta^{2}}{100},
$$

which is again always well below unity for objects of Table 5 .

The photon field can also originate outside the reconnection region. For example, $U_{\mathrm{ph}}$ can be evaluated at a distance $R$ from an object of mass $M$ emitting at a fraction $\alpha$ of the Eddington luminosity $L_{\mathrm{Edd}}=4 \pi G M m_{\mathrm{i}} c / \sigma_{\mathrm{T}}$ :

$$
\begin{aligned}
U_{\mathrm{ph}} & =\frac{\alpha L_{\mathrm{Edd}}}{4 \pi R^{2} c}=\alpha\left(\frac{R_{\mathrm{S}}}{R}\right)^{2} \frac{m_{\mathrm{i}} c^{2}}{2 \sigma_{\mathrm{T}} R_{\mathrm{S}}} \\
& =\alpha\left(\frac{R_{\mathrm{S}}}{R}\right)^{2} \frac{10 \mathrm{~km}}{R_{\mathrm{S}}} \times 10^{15} \mathrm{erg} / \mathrm{cm}^{3},
\end{aligned}
$$

with $R_{\mathrm{S}}$ the Schwarzschild radius of the object. With Eq. (A.3), it gives a loss per $\omega_{\text {pe }}^{-1}$ given by

$\frac{\delta E_{\mathrm{IC}, \mathrm{Edd}}}{\gamma m_{\mathrm{e}} c^{2}}=6 \times 10^{4} \times\left(\frac{1 \mathrm{~cm}^{-3}}{n_{\mathrm{e}}}\right)^{1 / 2} \frac{\gamma \beta^{2}}{100} \alpha\left(\frac{R_{\mathrm{S}}}{R}\right)^{2} \frac{10 \mathrm{~km}}{R_{\mathrm{S}}}$.

\section{High-energy photons and opacity to $\gamma \gamma$-annihilation}

The photons emitted by the accelerated electrons (of Lorentz factor $\gamma$, velocity $\beta c$ ) can be due either to synchrotron or to Bremsstrahlung radiation. In the synchrotron case, photon energies can reach

$$
\begin{aligned}
h v_{\text {sync }} & =\frac{3 \gamma^{2} \omega_{\mathrm{ce}}}{2 \pi \beta} \\
& =3.5 \times 10^{-4} \mathrm{eV} \frac{B}{1 \mathrm{G}}\left(\frac{\gamma}{100}\right)^{2} \\
& =6.8 \times 10^{-10} m_{\mathrm{e}} c^{2} \frac{B}{1 \mathrm{G}}\left(\frac{\gamma}{100}\right)^{2} .
\end{aligned}
$$

Also, a thermal Bremsstrahlung spectra from electrons at temperature $T_{\mathrm{e}}$ cuts off above

$h v_{\text {therm }}=m_{\mathrm{e}} c^{2} \frac{T_{\mathrm{e}}}{6 \times 10^{9} \mathrm{~K}}$.

High-energy photons can annihilate with lower energy photons of energy $\epsilon_{0}$ only if they have an energy above $m_{\mathrm{e}}^{2} c^{4} / \epsilon_{0}$ (Gould $\&$ Schréder 1967), which requires at least a high-energy photon above $0.5 \mathrm{MeV}$. Synchrotron radiation can produce such photons in pulsar wind nebulae, and thermal Bremsstrahlung can do so in microquasars and GRBs. Also, high-energy photons can be produced by inverse-Compton collisions between ambient photons of energy $\epsilon_{0}$ and high-energy electrons of Lorentz factor $\gamma$. The outcome of such a collision is a high-energy photon of energy up to $\gamma^{2} \epsilon_{0}$, so that $\gamma>m_{\mathrm{e}} c^{2} / \epsilon_{0}$ is needed to produce pairs.

It is then interesting to compute the mean-free-path $l_{\gamma \gamma}$ of such high-energy photons. Their annihilation creates pairs, which will affect the reconnection dynamics only if they are created near the reconnection site. The relevant quantity is thus $l_{\gamma \gamma} / d_{\mathrm{e}}$, with $d_{\mathrm{e}}$ the electron inertial length. We only seek an order-of-magnitude estimate. From Gould \& Schréder (1967), we can approximate the optical depth $\tau_{\gamma \gamma}$ for a high-energy photon (energy $E$ ) traveling a length $l$ through a gas of lower energy photons (with a typical energy $\epsilon_{0}$, of number density $n_{\mathrm{ph}}$ ) as

$\tau_{\gamma \gamma} \sim l \pi r_{0}^{2} n_{\mathrm{ph}} f\left(m_{\mathrm{e}}^{2} c^{4} /\left\{E \epsilon_{0}\right\}\right)$,

where $r_{0}$ is the classical electron radius, and $f$ a function depending on the exact gas photon distribution. Generally, $f$ is a maximum and equal to $\sim 1$ for $E=\epsilon_{0}$. For example, if the gas of photons is a blackbody at temperature $T_{\mathrm{ph}}$, then $\epsilon_{0}=T_{\mathrm{ph}}$, $n_{\mathrm{ph}}=2 \zeta(3) / \pi^{2}\left(T_{\mathrm{ph}} /(\hbar c)\right)^{3}$, with $\zeta(3) \sim 1.202$, and $f$ is at its maximum for $E=T_{\mathrm{ph}}$ with a value $1 / 2 \zeta(3)$. The mean-free-path is defined such that $\tau_{\gamma \gamma}=1$. In the blackbody case, we have

$\frac{l_{\gamma \gamma, \mathrm{BB}}}{d_{\mathrm{e}}} \sim \frac{1}{\pi r_{0}^{2} d_{\mathrm{e}} n_{\mathrm{ph}}}=\left(\frac{n_{\mathrm{e}}}{1 \mathrm{~cm}^{-3}}\right)^{1 / 2}\left(\frac{10^{6} \mathrm{~K}}{T_{\mathrm{ph}}}\right)^{3}$.

Consequently, if $T_{\mathrm{ph}}<10^{6} \mathrm{~K}$, then the $\gamma \gamma$ annihilations occur well outside the reconnection region and do not affect the process; while for higher photon temperatures, the annihilation occurs after a free flight of less than an inertial length, i.e., inside 
the reconnection region. For an unspecified photon number density, Eq. (A.14) can be written

$$
\frac{l_{\gamma \gamma}}{d_{\mathrm{e}}} \sim \frac{1}{\pi r_{0}^{2} d_{\mathrm{e}} n_{\mathrm{ph}}}=\left(\frac{n_{\mathrm{e}}}{1 \mathrm{~cm}^{-3}}\right)^{1 / 2} \frac{2 \times 10^{19} \mathrm{~cm}^{-3}}{n_{\mathrm{ph}}} .
$$

However, as previously noted, a blackbody radiation at $T_{\mathrm{ph}}$ requires the thermalization of the photons by the hot electrons, which is impossible to achieve if these electrons are confined to the reconnection region on inertial length scales (Eq. (A.4)). Again, in the optically thin case the radiation number density should be estimated as $n_{\mathrm{ph}} \sim a d_{\mathrm{e}} / c \times \mathrm{d} n_{\mathrm{ph}} / \mathrm{d} t$, with $\mathrm{d} n_{\mathrm{ph}} / \mathrm{d} t$ the production rate of photons and $\left(a d_{\mathrm{e}}\right)^{3}$ the volume of the emission region. We then evaluate $n_{\mathrm{ph}}$ for synchrotron and Bremsstrahlung radiation, and for the vicinity of an object emitting at a given luminosity.

For synchrotron radiation, this can be roughly estimated by dividing the total power emitted by an electron by the characteristic energy $h v_{\text {sync }}$ (Eq. (A.11)), and then multiplying by the electron number density. After some manipulations, one arrives at $\mathrm{d} n_{\mathrm{ph}} / \mathrm{d} t=P_{\text {emit }} /\left(h v_{\text {sync }}\right)=0.1 \omega_{\text {ce }}$, so that

$n_{\mathrm{ph}, \mathrm{sync}}=0.1 a \frac{\omega_{\mathrm{ce}}}{\omega_{\mathrm{pe}}} n_{\mathrm{e}}$,

with $\omega_{\text {pe }}$ the non-relativistic plasma frequency associated to $d_{\mathrm{e}}$. We can rewrite $\omega_{\text {ce }} / \omega_{\text {pe }}=\left(\sigma_{\mathrm{e}}^{\text {cold }}\right)^{1 / 2}$ with $\sigma_{\mathrm{e}}^{\text {cold }}=B^{2} /\left(\mu_{0} n_{\mathrm{e}} m_{\mathrm{e}} c^{2}\right)$. Inserting $n_{\mathrm{ph} \text {,sync }}$ into Eq. (A.15), we have

$\frac{l_{\gamma \gamma, \mathrm{sync}}}{d_{\mathrm{e}}} \sim a^{-1}\left(\frac{1 \mathrm{~cm}^{-3}}{n_{\mathrm{e}}}\right)^{1 / 2}\left(\frac{10^{10}}{\sigma_{\mathrm{e}}^{\text {cold }}}\right)^{1 / 2}$.

For radio lobes, radio-emitting regions of extragalactic jets or pulsar wind nebulae, $l_{\gamma \gamma, \text { sync }} \gg d_{\mathrm{e}}$ holds, so that pairs form far away from the reconnection site. For microquasar coronae close to the hole, for extragalactic jet $\gamma$-ray region, for GRB jets, or for pulsar wind termination shocks, we have $l_{\gamma \gamma \text {,sync }} \ll d_{\mathrm{e}}$ and pairs form inside the reconnection region.

For Bremsstrahlung radiation, one can estimate the photon number density by dividing the Bremsstrahlung emissivity $P_{\text {emit }}$ by the typical energy $T_{\mathrm{e}}$, and multiplying by a photon escape length $a d_{\mathrm{e}}$. We find

$n_{\mathrm{ph}, \mathrm{Brem}}=1.8 \times 10^{-20} \mathrm{~cm}^{-3} a\left(\frac{10^{8} \mathrm{~K}}{T_{\mathrm{e}}}\right)^{1 / 2}\left(\frac{n_{\mathrm{e}}}{1 \mathrm{~cm}^{-3}}\right)^{3 / 2}$.

Inserting into Eq. (A.15), we have

$\frac{l_{\gamma \gamma, \mathrm{Brem}}}{d_{\mathrm{e}}} \sim 10^{39} \times a^{-1}\left(\frac{1 \mathrm{~cm}^{-3}}{n_{\mathrm{e}}}\right)\left(\frac{T_{\mathrm{e}}}{10^{8} \mathrm{~K}}\right)^{1 / 2}$.

We have $l_{\gamma \gamma, \mathrm{Brem}} \gg d_{\mathrm{e}}$ for all objects of Table 5 .

For microquasars, the photon field number density can also be evaluated at a distance $R$ from a source emitting at $\alpha L_{\text {Edd }}$. To do so, we divide the energy density of Eq. (A.9) by a typical frequency $h v_{\text {typ }}$, to obtain

$n_{\mathrm{ph}}=\alpha\left(\frac{R_{\mathrm{S}}}{R}\right)^{2} \frac{10 \mathrm{~km}}{R_{\mathrm{S}}} \frac{1 \mathrm{keV}}{h v_{\mathrm{typ}}} \times 10^{24} \mathrm{~cm}^{-3}$.

Inserting into Eq. (A.15), we obtain

$$
\frac{l_{\gamma \gamma, \mathrm{Edd}}}{d_{\mathrm{e}}} \sim 2 \times 10^{-5}\left(\frac{n_{\mathrm{e}}}{1 \mathrm{~cm}^{-3}}\right)^{1 / 2} \alpha^{-1}\left(\frac{R}{R_{\mathrm{S}}}\right)^{2} \frac{R_{\mathrm{S}}}{10 \mathrm{~km}} \frac{h v_{\mathrm{typ}}}{1 \mathrm{keV}} .
$$

Consequently, close to the central object, for high luminosities and electron number densities that are not too high, pair creation will be very significant.

\section{References}

Atoyan, A. M., \& Aharonian, F. A. 1996, A\&AS, 120, C453

Aunai, N., Hesse, M., Zenitani, S., et al. 2013, Phys. Plasmas, 20, 022902

Baumann, G., \& Nordlund, Å. 2012, ApJ, 759, L9

Bednarek, W., \& Idec, W. 2011, MNRAS, 414, 2229

Bell, A. R. 1978, MNRAS, 182, 147

Bessho, N., \& Bhattacharjee, A. 2012, ApJ, 750, 129

Birk, G. T., Crusius-Wätzel, A. R., \& Lesch, H. 2001, ApJ, 559, 96

Bosch-Ramon, V. 2012, A\&A, 542, A125

Bucciantini, N., Arons, J., \& Amato, E. 2011, MNRAS, 410, 381

Cerutti, B., Uzdensky, D. A., \& Begelman, M. C. 2012a, ApJ, 746, 148

Cerutti, B., Werner, G. R., Uzdensky, D. A., \& Begelman, M. C. 2012b, ApJ, 754, L33

Cerutti, B., Werner, G. R., Uzdensky, D. A., \& Begelman, M. C. 2013, ApJ, 770, 147

Childs, H., Brugger, E., Whitlock, B., et al. 2012, in High Performance Visualization, Enabling Extreme-Scale Scientific Insight (Chapman \& Hall/CRC), 357

Daldorff, L. K. S., Tóth, G., Gombosi, T. I., et al. 2014, J. Comput. Phys., 268, 236

Daughton, W., Roytershteyn, V., Karimabadi, H., et al. 2011, Nature Phys., 7, 539

de Gouveia dal Pino, E. M., \& Lazarian, A. 2005, A\&A, 441, 845

de Gouveia Dal Pino, E. M., Piovezan, P. P., \& Kadowaki, L. H. S. 2010, A\&A, 518, A5

Del Santo, M., Malzac, J., Belmont, R., Bouchet, L., \& De Cesare, G. 2013, MNRAS, 430, 209

Dexter, J., McKinney, J. C., Markoff, S., \& Tchekhovskoy, A. 2014, MNRAS, 440,2185

Di Matteo, T. 1998, MNRAS, 299, L15

Di Matteo, T., Blackman, E. G., \& Fabian, A. C. 1997, MNRAS, 291, L23

Díaz, María, T., Miller-Jones, J. C. A., Migliari, S., Broderick, J. W., \& Tzioumis, T. 2013, Nature, 504, 260

Drake, J. F., Shay, M. A., Thongthai, W., \& Swisdak, M. 2005, Phys. Rev. Lett., 94, 095001

Drake, J. F., Swisdak, M., Che, H., \& Shay, M. A. 2006, Nature, 443, 553

Drake, J. F., Opher, M., Swisdak, M., \& Chamoun, J. N. 2010, ApJ, 709, 963

Drenkhahn, G., \& Spruit, H. C. 2002, A\&A, 391, 1141

Drury, L. O. 2012, MNRAS, 422, 2474

Eastwood, J. P., Phan, T. D., Øieroset, M., et al. 2013, Plasma Phys. Control. Fusion, 55, 124001

Egedal, J., Daughton, W., Drake, J. F., Katz, N., \& Lê, A. 2009, Phys. Plasmas, 16,050701

Egedal, J., Daughton, W., \& Le, A. 2012, Nature Phys., 8, 321

Giannios, D. 2010, MNRAS, 408, L46

Giannios, D., \& Spruit, H. C. 2007, A\&A, 469, 1

Giannios, D., Uzdensky, D. A., \& Begelman, M. C. 2009, MNRAS, 395, L29

Giroletti, M., Giovannini, G., Feretti, L., et al. 2004, ApJ, 600, 127

Goodman, J., \& Uzdensky, D. 2008, ApJ, 688, 555

Gould, R. J., \& Schréder, G. P. 1967, Phys. Rev., 155, 1404

Graf von der Pahlen, J., \& Tsiklauri, D. 2014, Phys. Plasmas, 21, 012901

Higashimori, K., \& Hoshino, M. 2012, J. Geophys. Res. (Space Phys.), 117, 1220

Hoshino, M. 2012, Phys. Rev. Lett., 108, 135003

IceCube Collaboration 2013, Science, 342, 1242856

Jaroschek, C. H., Treumann, R. A., Lesch, H., \& Scholer, M. 2004, Phys. Plasmas, 11, 1151

Jaroschek, C. H., Hoshino, M., Lesch, H., \& Treumann, R. A. 2008, Adv. Space Res., 41, 481

Ji, H., \& Daughton, W. 2011, Phys. Plasmas, 18, 111207

Jiang, Y.-F., Stone, J. M., \& Davis, S. W. 2014, ApJ, 784, 169

Kagan, D., Milosavljević, M., \& Spitkovsky, A. 2013, ApJ, 774, 41

Kato, T. N. 2013 [arXiv: 1312 . 5507]

Khotyaintsev, Y. V., Vaivads, A., Retinò, A., et al. 2006, Phys. Rev. Lett., 97, 205003

Kirk, J. G., \& Skjæraasen, O. 2003, ApJ, 591, 366

Klimas, A., Hesse, M., Zenitani, S., \& Kuznetsova, M. 2010, Phys. Plasmas, 17, 112904

Kotani, T., Kawai, N., Aoki, T., et al. 1994, PASJ, 46, L147

Kowal, G., de Gouveia Dal Pino, E. M., \& Lazarian, A. 2011, ApJ, 735, 102

Kronberg, P. P., Colgate, S. A., Li, H., \& Dufton, Q. W. 2004, ApJ, 604, L77

Lazar, A., Nakar, E., \& Piran, T. 2009, ApJ, 695, L10

Liu, W., Li, H., Yin, L., et al. 2011, Phys. Plasmas, 18, 052105

Liu, Y.-H., Drake, J. F., \& Swisdak, M. 2012, Phys. Plasmas, 19, 022110

Liu, Y.-H., Daughton, W., Karimabadi, H., Li, H., \& Peter Gary, S. 2014, Phys. Plasmas, 21, 022113

Lyubarsky, Y., \& Liverts, M. 2008, ApJ, 682, 1436 
Lyutikov, M. 2006a, MNRAS, 369, L5

Lyutikov, M. 2006b, MNRAS, 367, 1594

Markidis, S., Henri, P., Lapenta, G., et al. 2013, Phys. Plasmas, 20, 082105

May, J., Tonge, J., Ellis, I., et al. 2014, Phys. Plasmas, 21, 052703

McKinney, J. C., \& Uzdensky, D. A. 2012, MNRAS, 419, 573

McKinney, J. C., Tchekhovskoy, A., \& Blandford, R. D. 2012, MNRAS, 423, 3083

Melzani, M., Winisdoerffer, C., Walder, R., et al. 2013, A\&A, 558, A133

Melzani, M., Walder, R., Folini, D., \& Winisdoerffer, C. 2014a, Int. J. Mod. Phys. Conf. Ser., 28, 60194

Melzani, M., Walder, R., Folini, D., Winisdoerffer, C., \& Favre, J. M. 2014b, A\&A, 570, A111

Merloni, A., \& Fabian, A. C. 2001, MNRAS, 321, 549

Meyer, M., Horns, D., \& Zechlin, H.-S. 2010, A\&A, 523, A2

Ohtani, H., \& Horiuchi, R. 2009, Plasma Fusion Res., 4, 24

Olshevsky, V., Lapenta, G., \& Markidis, S. 2013, Phys. Rev. Lett., 111, 045002

Pei, W., Horiuchi, R., \& Sato, T. 2001, Phys. Plasmas, 8, 3251

Pétri, J., \& Lyubarsky, Y. 2007, A\&A, 473, 683

Petropoulou, M., Giannios, D., \& Dimitrakoudis, S. 2014, MNRAS, 445, 570

Pontin, D. I. 2011, Adv. Space Res., 47, 1508

Poutanen, J., \& Veledina, A. 2014, Space Sci. Rev., 183, 61
Pritchett, P. L. 2005a, J. Geophys. Res. (Space Phys.), 110, 5209

Pritchett, P. L. 2005b, J. Geophys. Res. (Space Phys.), 110, 10213

Reis, R. C., \& Miller, J. M. 2013, ApJ, 769, L7

Romanova, M. M., \& Lovelace, R. V. E. 1992, A\&A, 262, 26

Romero, G. E., Vieyro, F. L., \& Chaty, S. 2014, A\&A, 562, L7

Rybicki, G. B., \& Lightman, A. P. 1979, Radiative processes in astrophysics, eds. G. B. Rybicki, \& A. P. Lightman

Schwartz, D. A., Marshall, H. L., Lovell, J. E. J., et al. 2006, ApJ, 640, 592

Sironi, L., \& Spitkovsky, A. 2011a, ApJ, 741, 39

Sironi, L., \& Spitkovsky, A. 2011b, ApJ, 726, 75

Sironi, L., \& Spitkovsky, A. 2014, ApJ, 783, L21

Uzdensky, D. A. 2011, Space Sci. Rev., 160, 45

Uzdensky, D. A., Cerutti, B., \& Begelman, M. C. 2011, ApJ, 737, L40

Vapirev, A. E., Lapenta, G., Divin, A., et al. 2013, J. Geophys. Res. (Space Phys.), 118, 1435

Walder, R., Melzani, M., Folini, D., Winisdoerffer, C., \& Favre, J. M. 2014, ASTRONUM 2013, Conf. Proc., 488, 141

Zdziarski, A. A., Pjanka, P., Sikora, M., \& Stawarz, Ł. 2014, MNRAS, 442, 3243

Zenitani, S., \& Hoshino, M. 2001, ApJ, 562, L63

Zenitani, S., \& Hoshino, M. 2007, ApJ, 670, 702

Zenitani, S., \& Hoshino, M. 2008, ApJ, 677, 530 